\title{
Preclinical safety of human embryonic stem cell-derived oligodendrocyte progenitors supporting clinical trials in spinal cord injury
}

\begin{abstract}
Aim: To characterize the preclinical safety profile of a human embryonic stem cellderived oligodendrocyte progenitor cell therapy product (AST-OPC1) in support of its use as a treatment for spinal cord injury $(\mathrm{SCl})$. Materials \& methods: The phenotype and functional capacity of AST-OPC1 was characterized in vitro and in vivo. Safety and toxicology of AST-OPC1 administration was assessed in rodent models of thoracic SCI. Results: These results identify AST-OPC1 as an early-stage oligodendrocyte progenitor population capable of promoting neurite outgrowth in vitro and myelination in vivo. AST-OPC1 administration did not cause any adverse clinical observations, toxicities, allodynia or tumors. Conclusion: These results supported initiation of a Phase I clinical trial in patients with sensorimotor complete thoracic SCl.
\end{abstract}

Keywords: biodistribution $\bullet$ clinical trial $\bullet$ human embryonic stem cells $\bullet$ oligodendrocyte progenitors $\bullet$ preclinical safety $\bullet$ thoracic spinal cord injury $\bullet$ toxicology $\bullet$ tumorigenicity

There are multiple pathologies observed in spinal cord injury (SCI) due to the injury itself and subsequent secondary effects due to edema, hemorrhage and inflammation [1]. These pathologies include the severing of axons, demyelination, parenchymal cavitation and the production of ectopic tissue such as fibrous scar tissue, gliosis and dystrophic calcification $[2,3]$. The clinical effects of these pathologies often are severe and can include limb paralysis, uncontrolled muscle spasms, aberrant pain signaling, loss of bladder function and sexual dysfunction [4]. Oligodendrocytes, which provide both neurotrophic factor and myelination support for axons, are susceptible to cell death following SCI and thus are an important therapeutic target [5]. Replacement of the oligodendrocyte population could both support the remaining and damaged axons and also remyelinate axons to help restore electrical conduction and function [6].

AST-OPC1 (formerly GRNOPC1) is a population of early-stage oligodendrocyte progenitor cells (OPCs) that is differentiated from human embryonic stem cells (hESCs) [7] using the $\mathrm{H} 1$ cell line. Originally developed by Geron Corporation as GRNOPC1, the name of this cell therapy product was subsequently changed to AST-OPC1 to mark the acquisition of Geron's hESC technology by Asterias. Importantly, the AST-OPC1 differentiation process is commercially scalable, compatible with current Good Manufacturing Practices (cGMP), has been approved for early Phase clinical testing by the US FDA, and remains one of the only protocols to be independently replicated by multiple groups and using different hESC lines [8]. ASTOPC1 has been characterized by the expression of several molecules that are associated with early-stage OPCs, such as Nestin, NG2 and PDGFR- $\alpha$ [9]. AST-OPC1 cells are further characterized by their minimal or lack of expression of markers known to be present in other cell types, such as neurons (Tuj1), astrocytes (GFAP), endoderm (AFP), mesoderm (MSA) and hESCs (Oct4) [10,11].

Studies of AST-OPC1 in rodent models of SCI provide evidence of the therapeutic benefit of these cells when transplanted directly into the injured spinal cord approximately
Catherine A Priest ${ }^{1,2}$, Nathan C Manley $^{3}$, Jerrod Denham ${ }^{1,4}$, Edward D Wirth III ${ }^{1,3}$ \& Jane S Lebkowski*,1,3

'Geron Corporation, 149 Commonwealth St., Menlo Park, CA 94025, USA

${ }^{2}$ California Institute of Regenerative Medicine, 210 King St., San Francisco, CA 94107, USA

${ }^{3}$ Asterias Biotherapeutics Inc., 230 Constitution Drive, Menlo Park, CA 94025, USA

${ }^{4}$ Dark Horse Consulting, 1999 South Bascom Ave Suite 700, Campbell, CA 95008, USA

*Author for correspondence: jlebkowski@asteriasbio.com 
1 week after injury. Post-transplant, AST-OPC1 cells (in this case differentiated from H7 hESCs) exhibited long-term persistence, limited migration from the injection site, differentiation into mature oligodendrocytes and were associated with reduced parenchymal cavitation, increased remyelination and enhanced functional recovery [10]. Consistent with this, prior studies have shown that transplantation of analogous OPCs, including those derived from adult mouse brain or embryonic rat spinal cord, also remyelinate denuded axons and stimulate improved locomotion and reflex function in both mouse and rat models [12-14]. In addition, AST-OPC1 was found to secrete multiple factors relevant to neural repair and supported neurite extension from sensory neurons in vitro [11].

In this report, we describe additional phenotypic and functional characterization of AST-OPC1 and present the design of, and results from, the preclinical safety studies that were submitted to the FDA as part of an Investigational New Drug Application. The cells used in this study were manufactured using the same differentiation procedure as prior studies, including those demonstrating efficacy of AST-OPC1 [10]. To obtain safety data that are relevant to both AST$\mathrm{OPC} 1$ and to its proposed clinical use, we conducted long-term safety and toxicology studies in a rat model of thoracic spinal cord contusion injury. These studies were designed to address key safety parameters associated with direct administration of AST-OPC1 into the injured spinal cord, including: biodistribution and long-term persistence of the transplanted cells; induction of any local or systemic toxicities; and ASTOPC1's capacity for tumor or ectopic tissue formation. To further understand how the tumorigenicity of AST-OPC1 might depend on a residual population of hESCs, we performed additional studies with immunodeficient mice that were administered AST-OPC1 spiked with increasing levels of hESCs. The results of these studies supported clearance by the FDA to initiate a Phase I clinical trial to assess safety of AST-OPC1 in patients with subacute, sensorimotor complete thoracic SCI.

\section{Materials \& methods}

Please see Supplementary Materials \& Methods for additional details of methodology.

\section{Animal subjects}

All procedures used in this study were approved by a board-certified veterinarian and the Geron IACUC committee and were conducted in accordance with the NIH Guide for the Care and Use of Laboratory Animals. Adult athymic nude rats (strain Crl:NIHFoxn $1^{r n u}$ ) were obtained from Charles Rivers Laborato- ries (MA, USA). Rag2 $2^{-1-} \gamma^{-1-}$ mice and shiverer- mice were obtained from Jackson Laboratories (ME, USA) and bred in-house to generate $\mathrm{Rag} 2^{-1-} \gamma \mathrm{c}^{-{ }^{-l}} / \mathrm{shi}^{-/-}$homozygous mice. All animal subjects were housed in standard conditions with a 12-h light/dark cycle, were provided food and water ad libitum, and were allowed to acclimate for a minimum of 1 week prior to surgery.

\section{Differentiation of AST-OPC1 from hESCs}

The WA01 (H1) hESC line was expanded in feederfree conditions $[15,16]$ and differentiated into ASTOPC1 according to published methods [7,17]. Briefly, hESC colonies were lifted with collagenase and manual scraping and then seeded into ultra low attachment flasks (Corning, Inc., NY, USA) in 50\% hESC growth medium and $50 \%$ glial progenitor medium (GPM) containing $4 \mathrm{ng} / \mathrm{ml}$ of bFGF and $20 \mathrm{ng} / \mathrm{ml}$ EGF to stimulate embryoid body formation. On day 1 post-seeding, medium was replaced with $50 \%$ hESC growth medium $/ 50 \%$ GPM containing $20 \mathrm{ng} / \mathrm{ml} \mathrm{EGF}$ and $10 \mu \mathrm{M}$ all-trans-retinoic acid (RA). On days 2-8, medium was replaced daily with $100 \%$ GPM containing $20 \mathrm{ng} / \mathrm{ml}$ EGF and $10 \mu \mathrm{M} \mathrm{RA}$. On days 9-26, embryoid bodies were maintained in GPM/EGF without RA, which was replaced every 2 days. On day 27, embryoid bodies were plated in flasks precoated with Matrigel (Corning, Inc.) and cultured in GPM/EGF for 7 days with medium exchange every 2 days. On day 34 , cells were harvested with trypsin, replated in Matrigel-coated flasks and cultured for an additional 7 days in GPM/EGF, with medium exchange every 2 days. On day 41, cells were harvested with trypsin, filtered to remove residual cell aggregates and cryopreserved in liquid nitrogen. All cultures were tested regularly for sterility and mycoplasma, and no contamination was detected. Prior to in vitro and in vivo testing, ASTOPC1 was thawed and washed in HBSS to remove cryopreservation medium. All studies were performed using cryopreserved and thawed AST-OPC1 cells, in accordance with their use in the subsequent thoracic SCI clinical trial.

\section{Analysis of differentiated AST-OPC1 by flow cytometry}

Differentiated AST-OPC1 samples were assayed for the presence of surface and intracellular markers using standard flow cytometry. For surface marker staining, day 41 AST-OPC1 samples were blocked and incubated with primary antibody and/or isotype control (NG2, cat \# 37-2300, Invitrogen, CA, USA; Mouse IgG1, cat \# 554121, BD Biosciences, CA, USA) followed by fluorescent-conjugated secondary antibodies. For intracellular marker staining, day 41 AST-OPC1 samples were tagged with ethidium monoazide (cat \# E2028, 
Sigma, MO, USA) for dead cell discrimination, followed by fixation using $2 \%$ paraformaldehyde (PFA) and then permeabilization with cold $90 \%$ methanol. The cells were blocked, and then incubated with primary antibody and/or isotype control (Oct4, cat \# SC8629, Santa Cruz, CA, USA; Goat IgG, cat \# SC2028; Santa Cruz; Nestin, cat \# MAB5326, Millipore, MA, USA; Mouse IgG1, cat \# 554121, BD Biosciences) followed by fluorescent-conjugated secondary antibodies. All samples were then acquired and the data analyzed on the BD Biosciences FACSCalibur ${ }^{\mathrm{TM}}$ cytometer system using Cellquest Pro software (BD Biosciences).

\section{Quantification of secreted factors in AST-OPC1 conditioned medium by Luminex}

Conditioned media from seven different AST-OPC1 lots was collected at the time of harvest (immediately prior to cryopreservation) and sent to AssayGate, Inc. (MD, USA) for Luminex-based detection of 66 secreted factors. Secreted factors that were detected in all seven lots and found to have putative roles in neural repair are reported.

\section{In vitro neurite outgrowth assay}

Embryonic day 18 Sprague-Dawley rat cortical cells were seeded at 80,000 cells $/ 0.5 \mathrm{ml} /$ well into poly-Llysine-coated 24-well plates and cultured in Neurobasal medium. On day 4 , each well received a $0.4 \mu \mathrm{M}$ pore Transwell insert containing either 200,000 ASTOPC1 cells in Neurobasal medium or Neurobasal medium alone.

On day 14, primary cortical neurons were fixed and stained with the anti-neurofilament antibody, SMI312 (ca \# ab24574, Abcam, Cambridge, UK) and fluorescent-conjugated secondary antibody. Stained plates were imaged on an INCell Analyzer 2000 (GE Healthcare, PA, USA), using a $10 \times$ objective to acquire nine imaging fields per well. ImageJ software (NIH, MD, USA) was used to determine the mean fluorescent area of SMI-312 positivity for each treatment group.

\section{Thoracic spinal cord injury}

Following at least 1 week of acclimation, adult male and female athymic nude rats (strain $=$ Crl:NIH-Foxn $1^{\mathrm{rnu}}$, age $=8-12$ weeks) were subjected to a thoracic spinal cord crush/contusion injury at level T10. Rats were given an intraperitoneal injection of a $60 \mathrm{mg} / \mathrm{kg}$ ketamine $/ 7.5 \mathrm{mg} / \mathrm{kg}$ xylazine cocktail to induce anesthesia, and then a midline skin incision was made at the T8-T11 level of the thoracic spinal cord. The paravertebral muscles were dissected bilaterally to visualize the transverse apophyses. A laminectomy was performed at T10 only, and a midline contusion injury was induced using the Infinite Horizons Impactor
(Precision Systems \& Instrumentation, VA, USA) set to deliver a 200 kdyne force impact. The impact force was recorded and animals that exhibited no deficit or severe deficit (i.e., inability to right self or self-feed) were excluded from studies ( $<5 \%$ of animals). Following contusion injury and wound closure, animals were given a subcutaneous (sc.) injection of lactated Ringer's solution $(10 \mathrm{ml})$ and maintained on an isothermic pad until recovery from anesthesia. Following contusion injury, manual bladder expression was performed twoto three-times daily for each animal until voluntary bladder expression returned.

\section{AST-OPC1 transplantation in injured nude rats \& Shiverer/Rag2 or SCID/Bg mice}

For athymic nude rats (Crl:NIH-Foxn1rnu), ASTOPC1 was injected into the spinal cord $6-8$ days postcontusion injury. Animals were positioned in a stereotaxic frame, and a $50 \mu \mathrm{l}$ Hamilton syringe outfitted with a 32 gauge needle (1-inch-long, 30 beveled tip) was used to deliver vehicle (Hank's Balanced Salt Solution, HBSS) or AST-OPC1 at $2.4 \times 10^{6}$ or $2.4 \times 10^{5}$ cells $/$ rat into the dorsal spinal parenchyma adjacent to the contusion epicenter via four injections of $6 \mu$ (high-dose AST-OPC1 or HBSS) or a single $2.4 \mu \mathrm{l}$ injection (lowdose AST-OPC1). For high-dose AST-OPC1, the four injections were positioned around the perimeter of the injury epicenter. For low-dose AST-OPC1, the single injection was positioned cranial and adjacent to the injury epicenter. All injections were administered approximately $1 \mathrm{~mm}$ ventral to the pial surface of the spinal cord. Cells or vehicle were administered at a rate of approximately $3 \mu \mathrm{l} / \mathrm{min}$.

The immunosuppressive article, anti-asialo GM1 antibody (GM1Ab, cat \# 986-10001, Wako Chemicals, VA, USA) was administered via an intraperitoneal injection to all athymic nude rats (vehicle and AST-OPC1 groups) 2 days prior to transplant surgery, on the day of transplantation, and 2 days after transplantation, and weekly thereafter as $1 \mathrm{mg} /$ injection in $0.2 \mathrm{ml}$ USP sterile saline for the study duration.

For Rag2 ${ }^{-1-} \gamma^{-1 /} / \mathrm{shi}^{-1-}$ (shiverer) mice, AST-OPC1 was injected into the uninjured spinal cord of adult male mice at T9-T10 at a dose ranging from $2.5 \times 10^{5}$ to $1 \times 10^{6}$ cells/mouse and at a concentration of $1 \times 10^{5} \mathrm{cell} / \mathrm{s} / \mu \mathrm{l}$ using the same approach as described for athymic nude rats. No additional immunosuppressive agents were given to mice used in these studies.

\section{Animal perfusion \& tissue processing for histology}

Central and peripheral tissues were collected at autopsy and immersion fixed in $10 \%$ formalin for paraffinembedding. For spinal cord, the approximate rostral 
and caudal extent of the affected spinal cord tissue, corresponding to approximately $1 \mathrm{~cm}$ rostral and $1 \mathrm{~cm}$ caudal to the contusion epicenter or site of administration, was dissected en bloc during necropsy. The tissue was processed for paraffin embedding using standard procedures. Spinal cord tissue was sectioned in the longitudinal/horizontal plane by microtome and $5 \mu \mathrm{m}$ sections were obtained and mounted onto slides for subsequent hematoxylin and eosin staining, ISH and immunohistochemistry (IHC).

Whole body fixation was performed by transcardial perfusion with $0.9 \%$ saline followed by $4 \%$ PFA. For frozen tissue preparation, perfused tissue was postfixed in $4 \%$ PFA overnight at $4^{\circ} \mathrm{C}$ followed by cryoprotection in $30 \%$ sucrose/PBS for $72 \mathrm{~h}$ at $4^{\circ} \mathrm{C}$. Cryoprotected tissue was snap-frozen on dry ice and stored at $-80^{\circ} \mathrm{C}$ until sectioning at $20 \mu \mathrm{m}$ on a cryostat.

\section{Myelin staining with Eriochrome cyanine}

To identify myelinated fibers within the spinal cord, tissues were stained with Eriochrome cyanine solution and counterstained with Eosin-Y. Stained slides were imaged with an Axiocam MRc5 camera mounted on an Observer D1 microscope (Carl Zeiss, Gottingen, Germany).

\section{Characterization of transplanted AST-OPC1 by immunohistochemistry \& in situ hybridization}

All animals that received AST-OPC1 transplantation were assayed for the presence of human cells in the spinal cord by in situ hybridization (ISH) with an human Alu DNA repeat sequence probe (cat \# Q151P.9900, Life Technologies, MA, USA) for paraffin-embedded tissue or IHC with an antihuman nuclei antibody (hNUC, cat \# MAB1281, Millipore) for frozen tissue. Sections were also stained by IHC for Ki-67 (cat \# ab833, Abcam).

\section{Cavitation area measurements in the injured spinal cord}

Measurements of cavity formation were performed on spinal cord tissues sectioned in the longitudinal (horizontal) plane and stained with Eriochrome cyanine/Eosin-Y as described above. For each animal included in the cavitation area analysis, all longitudinal sections containing the injury site were examined, and the section containing the maximal cavitation area was selected and used for quantification. Measurements were made on spinal cord sections from ten to 12 subjects in each treatment group $\left(2.4 \times 10^{5}\right.$ AST-OPC1, $2.4 \times 10^{6}$ AST-OPC1 or HBSS). Subjects within each treatment group were selected randomly, and area measurements were performed using ImageJ software by an investigator who was blinded to the treatment groups.

\section{Biodistribution of transplanted AST-OPC1 by quantitative PCR}

One week after thoracic spinal cord injury, rats were transplanted with AST-OPC1 or vehicle control, using the methods described above and two different doses of AST-OPC1 $\left(2.4 \times 10^{5}\right.$ or $2.4 \times 10^{6}$ cells $/$ rat $)$. At 2 , 14 and 180 days post-administration, blood, cerebral spinal fluid (CSF) and tissues were collected from the transplanted rats. Tissues and CSF were flash frozen in isopentane chilled on dry ice and stored at $-80^{\circ} \mathrm{C}$. The presence of human DNA was assayed by amplifying a 232 base pair sequence of the human Alu Y repeat sequence using the ABI Prism 7700 Sequence Detection System. All quantitative PCR (qPCR) analyses were performed by Althea Technologies (CA, USA).

At 2, 14 and 180 days after AST-OPC1 transplantation, parallel sets of animals in each group were transcardially perfused using $0.9 \%$ saline followed by $4 \%$ PFA, and the spinal cord and brain were sectioned on a cryostat for subsequent detection of human cells by IHC with anti-hNUC.

\section{Clinical \& toxicological assessments of AST-OPC1-treated, contused rats}

Toxicology studies of AST-OPC1-treated, contused male and female athymic nude rats were performed at MPI Research (MI, USA) using GLP. Observations for morbidity, mortality, injury and the availability of food and water were conducted at least twice daily for all animals enrolled in toxicology studies. Clinical observations were conducted and bodyweights were measured and recorded twice weekly during the study. At study termination, necropsy examinations were performed, organ weights were recorded, blood and urine samples were collected for clinical pathology evaluations and selected tissues were examined microscopically by a board-certified veterinary pathologist who was blinded to animals' treatment groups.

\section{Allodynia measurements in AST-OPC1-treated, contused rats}

At approximately 3, 6 and 9 months post-transplantation, ten male and ten female rats from each treatment group $\left(2.4 \times 10^{5} \mathrm{AST}\right.$-OPC1, $2.4 \times 10^{6} \mathrm{AST}$-OPC1 or HBSS) were evaluated for allodynia or hypersensitivity in response to normally non-noxious mechanical (blunt probe) or cold (point application of acetone) stimuli [18]. Each animal tested was observed for supraspinal responses to the stimuli according to the following parameters: three anatomical levels of assessment (at, above, and below the level of injury), two modalities (application of mechanical stimulation or cold stimulation) and two assay sites (dorsal skin surface of the trunk and glabrous tissue of the paws). 


\section{Mouse tumorigenicity studies of AST-OPC1 spiked with undifferentiated hESCs}

Tumorigenicity studies were performed at MPI Research using GLP. AST-OPC1 spiked with its parent hESC line, H1, was administered into the thoracic spinal cord of uninjured male and female CB-17/Icr$\mathrm{Crl}-P r k d c^{\text {sid }}$ Lyst ${ }^{b g} \mathrm{BR}$ (SCID/bg) mice using the transplantation procedure described above. Animals were administered a total dose of $2 \times 10^{6}$ cells or HBSS as a single $10 \mu \mathrm{l}$ injection and were monitored for up to 12 months for clinical signs of tumor formation: treatment groups and sample sizes were as follows: $100 \%$ $\mathrm{H} 1, \mathrm{n}=30 ; 50 \% \mathrm{H} 1, \mathrm{n}=30 ; 10 \% \mathrm{H} 1, \mathrm{n}=31 ; 5 \% \mathrm{H} 1$, $\mathrm{n}=12 ; 1 \% \mathrm{H} 1, \mathrm{n}=36 ; 100 \%$ AST-OPC1, $\mathrm{n}=128$; HBSS, $\mathrm{n}=30$. The sample size for the $0 \%$ hESC group (AST-OPC1 alone) was selected such that if all animals in that group were negative for tumors, the true incidence of tumor formation would be $<3 \%(3 / \mathrm{n})$ at $90 \%$ confidence. Sample sizes were chosen to allow for detection of a teratoma frequency $\geq 3 \%$ for the 1,10 , 50 and $100 \%$ hESC spiked groups. Microscopic examination of fixed hematoxylin and eosin-stained paraffin sections was performed on protocol-designated sections of tissues. The slides were examined by a board-certified veterinary pathologist who was blinded to the treatment groups. A four-step grading system was utilized to define gradable lesions for comparison between treatment groups. Presence of human cells in observed tumors was confirmed by ISH as described above.

\section{Results}

\section{Description of AST-OPC1}

AST-OPC1 (formerly known as GRNOPC1) was generated by the differentiation of WA01 (H1) hESCs from a master cell bank as described in the Supplementary Methods. The differentiation process to produce AST-OPC1 requires 41 days and transitions the hESCs from undifferentiated cell colonies through embryoid bodies to become an adherent, dispersed cell population which is harvested and cryopreserved. Representative photomicrographs of the cells at different stages of the 41-day differentiation process are shown in Figure $1 \mathrm{~A}$.

Analysis of 41-day differentiated AST-OPC1 by flow cytometry and immunocytochemistry (ICC) indicated that the cell population was comprised mostly of neural lineage cells with a phenotype consistent with preOPCs to early OPCs. By flow cytometry, over $90 \%$ of the cells were positive for Nestin and $>50 \%$ were positive for NG2, a neural/glial proteoglycan expressed by oligodendrocyte progenitor cells (Figure 1B). In addition, levels of the pluripotent stem cell marker, Oct4, were below the level of detection $(<0.2 \%)$, indicat- ing little to no residual hESCs (Figure 1B). Using the AST-OPC1 differentiation process, we produced over 75 lots of AST-OPC1, which were further characterized by ICC on day 41 for the presence of multiple markers of ectodermal, mesodermal, endodermal and pluripotent cell types to assess the composition of the population and detect potential unwanted cells types (Supplementary Table 1). In agreement with the flow cytometry results, ICC profiling indicated a cell population predominantly composed of pre-OPCs or early OPCs with few mature neuronal or astrocytic cells. The presence of endodermal, mesodermal or pluripotent cell types ranged from undetectable to $<1 \%$ of the differentiated AST-OPC1 cell population. The data shown in Figure 1 represent a typical lot of AST-OPC1 that can be further defined as a population $>90 \%$ Nestin positive, $30-70 \%$ NG2 positive and $<0.2 \%$ Oct 4 positive. The lots of AST-OPC1 used for the preclinical studies described in this manuscript were selected based on these criteria.

\section{Biological activities of AST-OPC1}

AST-OPC1 stimulated axonal outgrowth in vitro and myelination in vivo. A noncontact co-culture system using rat primary cortical neurons was used to assess the ability of AST-OPC1 to induce axonal outgrowth in vitro via paracrine signaling. Figure 2 shows rat primary cortical neurons that were cultured 14 days in control medium (Figure 2A) or with ASTOPC1 (Figure 2B) and labeled by ICC with antibodies against the axonal marker SMI-312. Quantitative enumeration of the area of axonal outgrowth from three independent experiments demonstrated significantly greater axonal outgrowth with AST-OPC1 co-culture (Figure $2 \mathrm{C}$ ). To identify candidate paracrine factors mediating this effect, proteins secreted by AST-OPC1 were characterized by Luminex assay. Expanding on previous findings [11], AST-OPC1 conditioned medium contained multiple proteins with putative roles in neural repair, including those involved in axonal growth (clusterin), chemoattraction (MCP-1) and suppression of apoptosis (TIMP1 and 2, ApoE) (Supplementary Table 2) [19-24].

To assess the ability of AST-OPC1 to induce myelination of axons, cryopreserved AST-OPC1 cells were thawed and injected into the spinal cord of immunodeficient Rag2 $2^{-1-} \gamma^{-1 /} / \mathrm{shi}^{-1-}$ (shiverer) mice that displayed a dysmyelinated phenotype due to their deficiency in myelin basic protein production. Two doses of AST-OPC1 $\left(2.5 \times 10^{5}\right.$ or $1 \times 10^{6}$ cells $)$ were injected into the uninjured spinal cord at thoracic level T9-T10. The mice were assessed 2 months postimplant for the presence of human cells and myelinated axons. Two months after implantation, human cells 
(A)

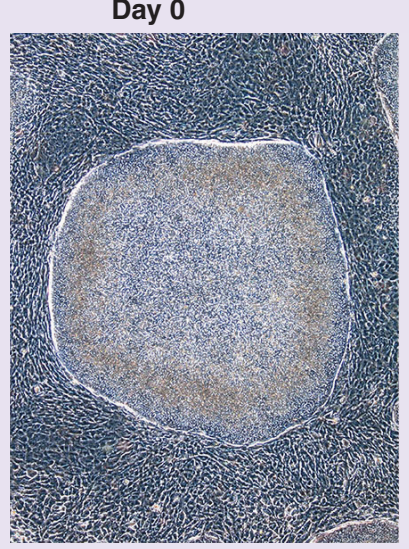

Day 17

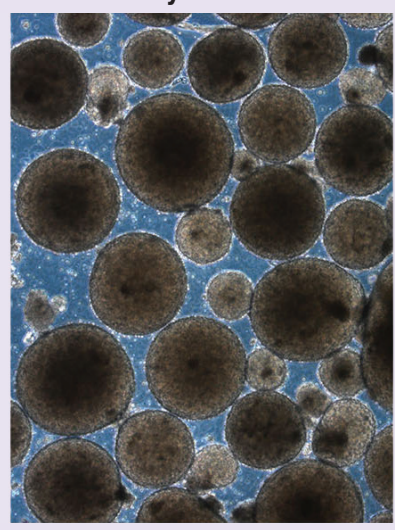

Day 34

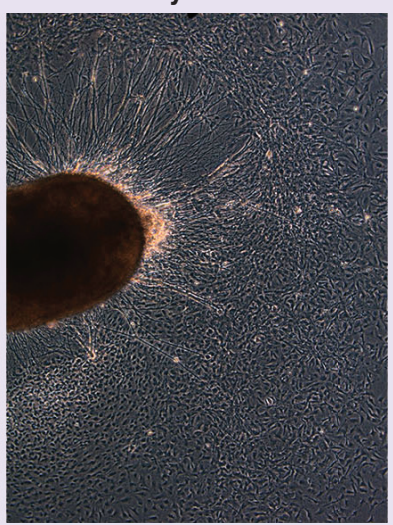

Day 41

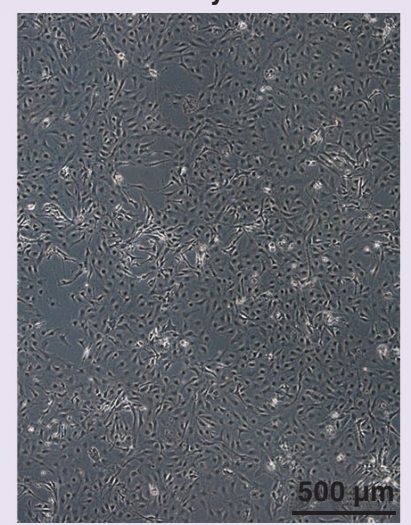

(B)

Day 41 Nestin
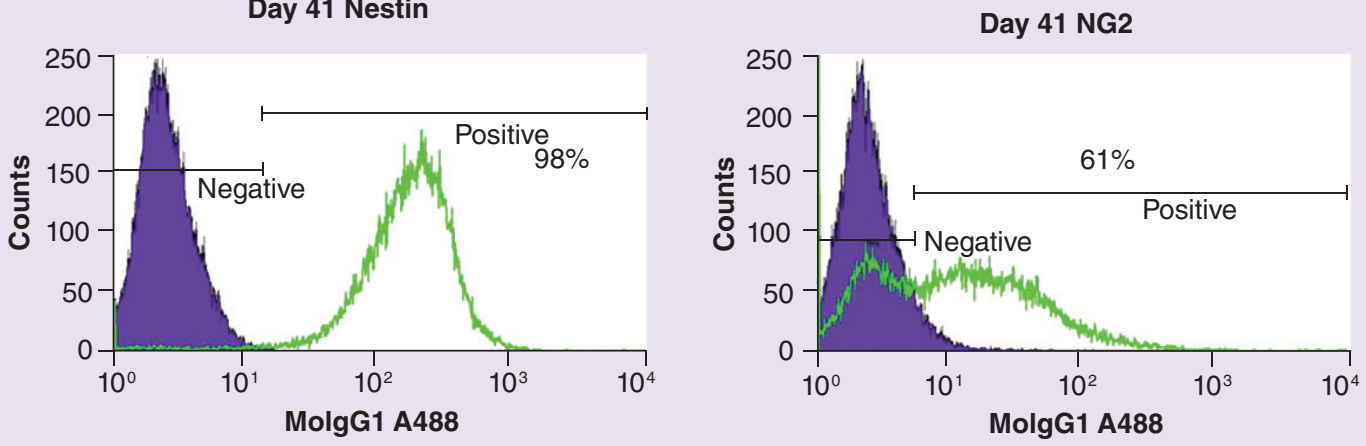

Day 41 Oct4

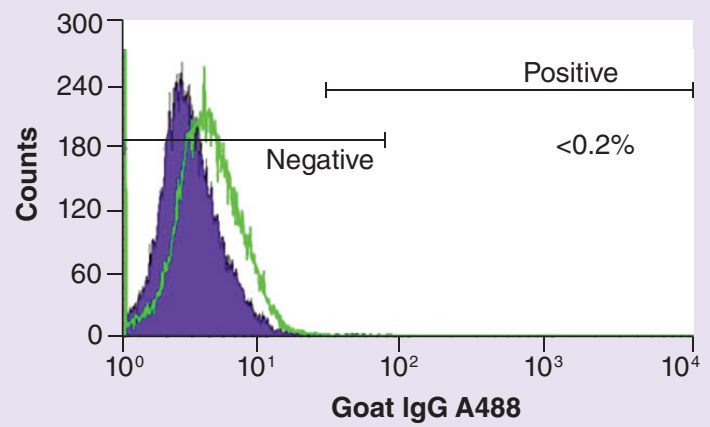

Figure 1. Differentiation of human embryonic stem cells into AST-OPC1. (A) Representative brightfield photomicrographs of the AST-OPC1 differentiation process. Undifferentiated human embryonic stem cells (day 0 ) undergo embryoid body formation and differentiation in suspension culture (days 1-26) followed by plating and additional differentiation and expansion as an adherent culture (days 27-41). Magnification = 50x. (B) Representative flow cytometry analysis of day 41 AST-OPC1 for the neural/glial lineage markers Nestin and NG2, and the pluripotency marker Oct4. Oct4 levels in AST-OPC1 were below the lower limit of detection by flow cytometry $(0.2 \%)$.

were detected by IHC using a human nuclear antigen antibody (hNUC, brown) in close association with myelinated fibers labeled with Eriochrome cyanine (EC, blue) within the thoracic spinal cord (Figure 2D). No myelinated fibers or human cells were observed outside the vicinity of the graft site (Figure 2E).

In rats subjected to thoracic SCI prior to ASTOPC1 administration, AST-OPC1 survived in the lesion site, was associated with myelinated fibers and reduced cavity formation. In a series of three toxicology studies, athymic nude rats [Crl:NIH-Foxn $1^{\text {rnu}}$ ] received a 200 kdyne contusion injury at T10 using the Infinite Horizon Impactor (Precision Systems \& Instrumentation) and were implanted with $2.4 \times 10^{5}$ or $2.4 \times 10^{6}$ AST-OPC1 or HBSS vehicle control at 6-9 days postinjury. 9 months postimplantation, the lesion sites were examined histologically. In control injured rats injected with vehicle, extensive paren- 
chymal cavitation was observed (Figure 3A \& C). The cavity was often large, extending rostrocaudally across multiple spinal cord segments from the epicenter of the injury site. Little to no cellularity existed in the cavity. Myelinated axons approached the injury cavity and halted their progression as evidenced by the presence of dystrophic endbulbs, or changed path to circumvent the contusion cavity. By contrast, injured animals injected with AST-OPC1 showed much less cavitation (Figure 3B \& D). In sections from the AST-OPC1 transplanted animals, multiple fascicles of myelinated axons stained with EC were frequently visible within the injury/graft site. In the largest toxicology study, paren- chymal cavitation was quantified for a subset of animals. In control animals injected with HBSS vehicle, measurable cavitation at the injury site was observed in 11 out of 12 subjects (Figure 3E). By contrast, rats injected with AST-OPC1, regardless of dose, exhibited a significant reduction in parenchymal cavitation with 12 out of 21 AST-OPC1-treated animals showing no injury-related cavities (Figure $3 \mathrm{E}$ ). Within the lesion site of AST-OPC1 treated animals, positive labeling with a human-specific Alu DNA repeat sequence probe (hAlu) using ISH confirmed that human cells were resident in the lesion site in the area of the myelinated fibers at 9 months post-implant (Figure 3F).
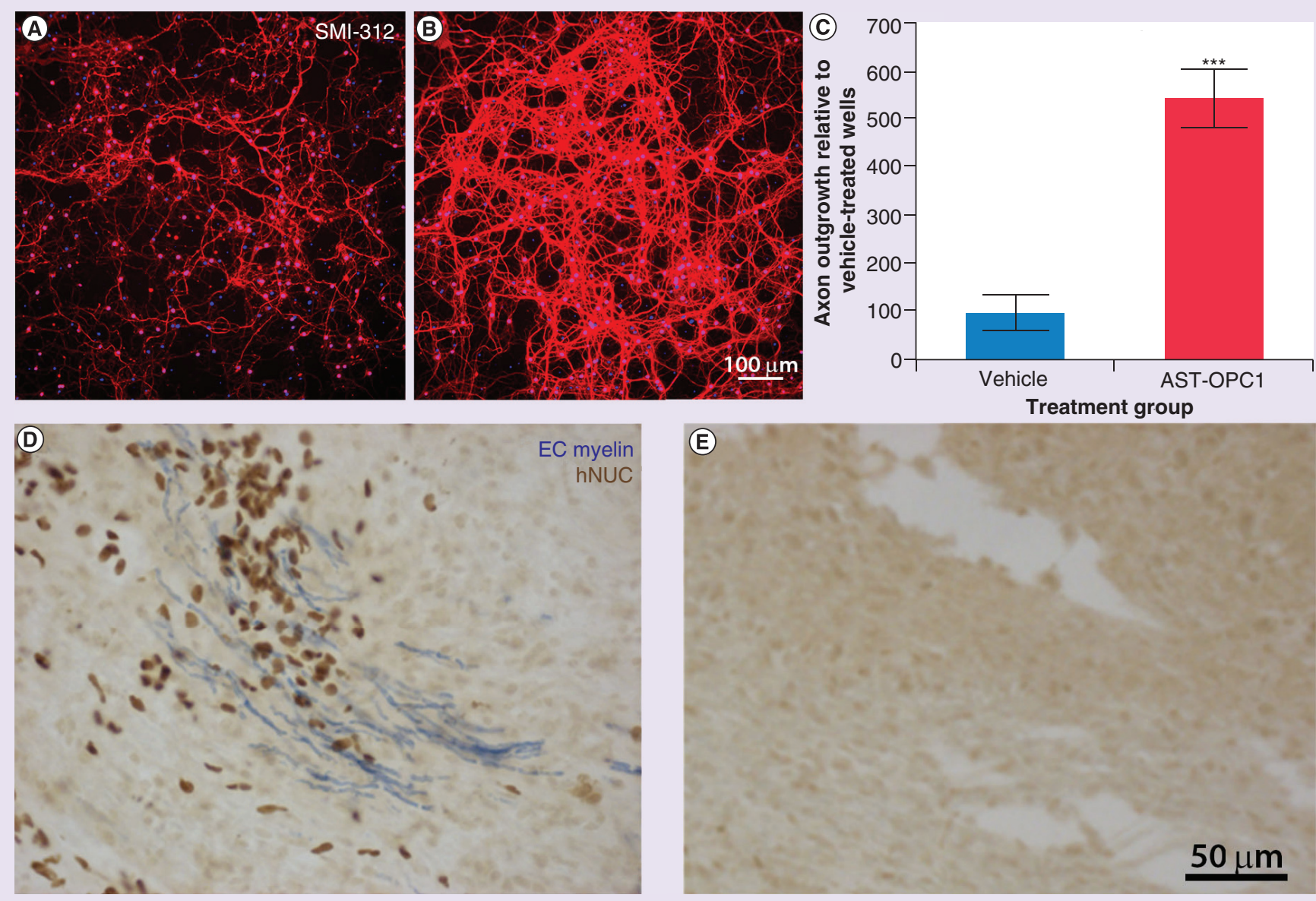

Treatment group

Figure 2. AST-OPC1 stimulates axon outgrowth in vitro and myelination in vivo. (A \& B) Representative images of rat primary cortical neurons cultured in control medium for 14 days (A) or co-cultured with AST-OPC1 from days 4-14 (B) and labeled by immunocytochemistry with antibodies against the axonal marker SMI-312. (C) Axon outgrowth was increased in rat primary cortical neurons co-cultured with AST-OPC1 compared with vehicle control on day 14 . The graph shows combined results from three independent experiments, expressed as average percent axon outgrowth relative to vehicle. *** indicates significance relative to vehicle by two-tailed student's $t$-test $(p<0.0001)$. Error bars denote standard deviation. (D \& E) High magnification photomicrographs of thoracic spinal cord from immunodeficient Rag ${ }^{-1-} \mathrm{yc}^{-1 /} / \mathrm{shi}^{-1-}$ mice transplanted with AST-OPC1. By 2 months post-transplantation, myelinated fibers (blue) were detectable within the graft site in close association with cells positively labeled with the anti-human nuclei antibody (hNUC, brown) (D), whereas no myelin staining was observed in the spinal cord outside the AST-OPC1 graft site (E). In this study, $7 \mathrm{Rag}^{-1-} \mathrm{\gamma C}^{-1 /} /$ shi mice were transplanted with AST-OPC1 and assessed at 2 months, positive hNUC staining was detectable in all subjects, and myelinated fibers were observed within the graft site in three of seven animals. Magnification: $($ A \& B) = 100x; D and $E=400 x$. 

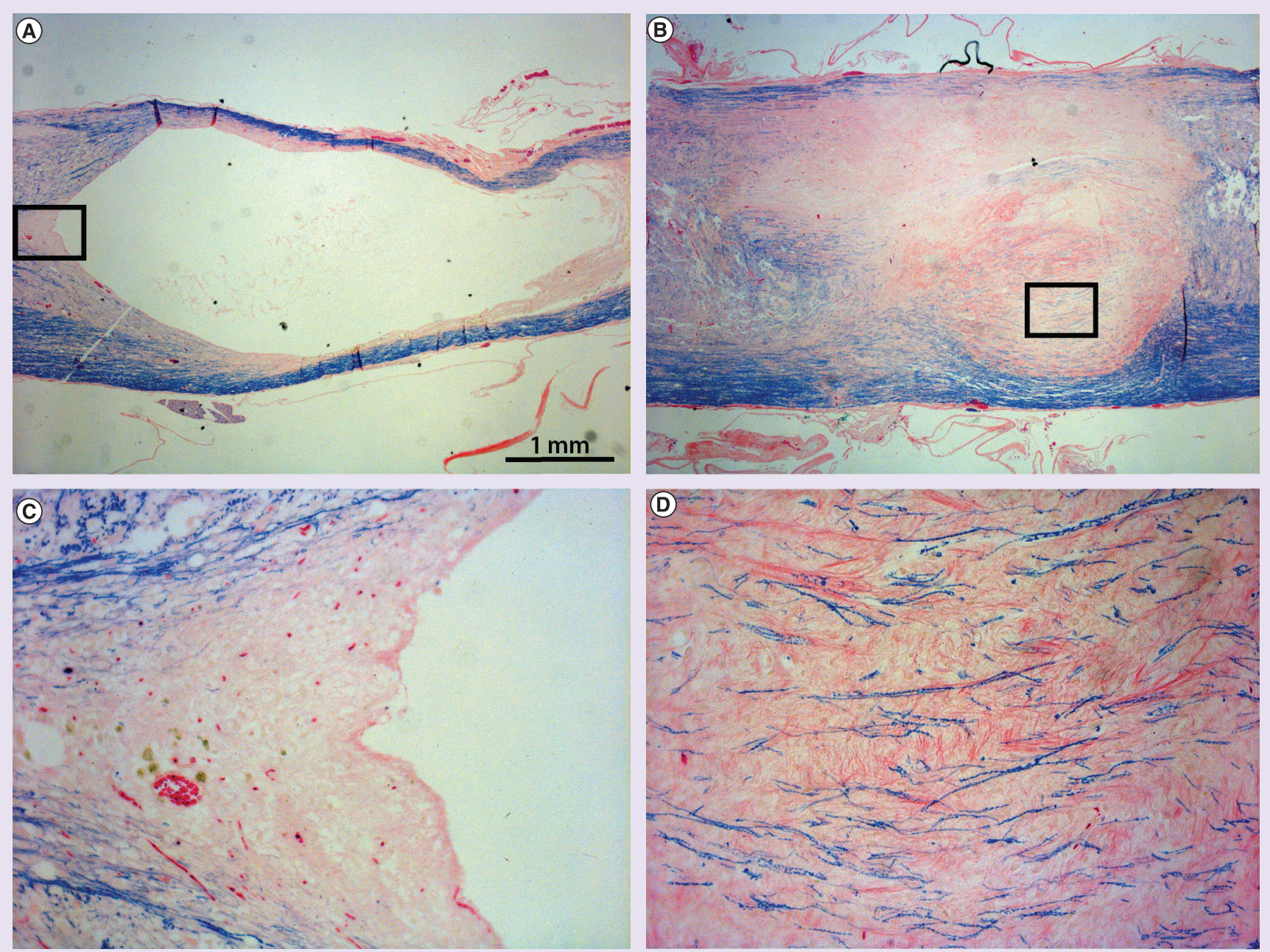

\section{(E)}
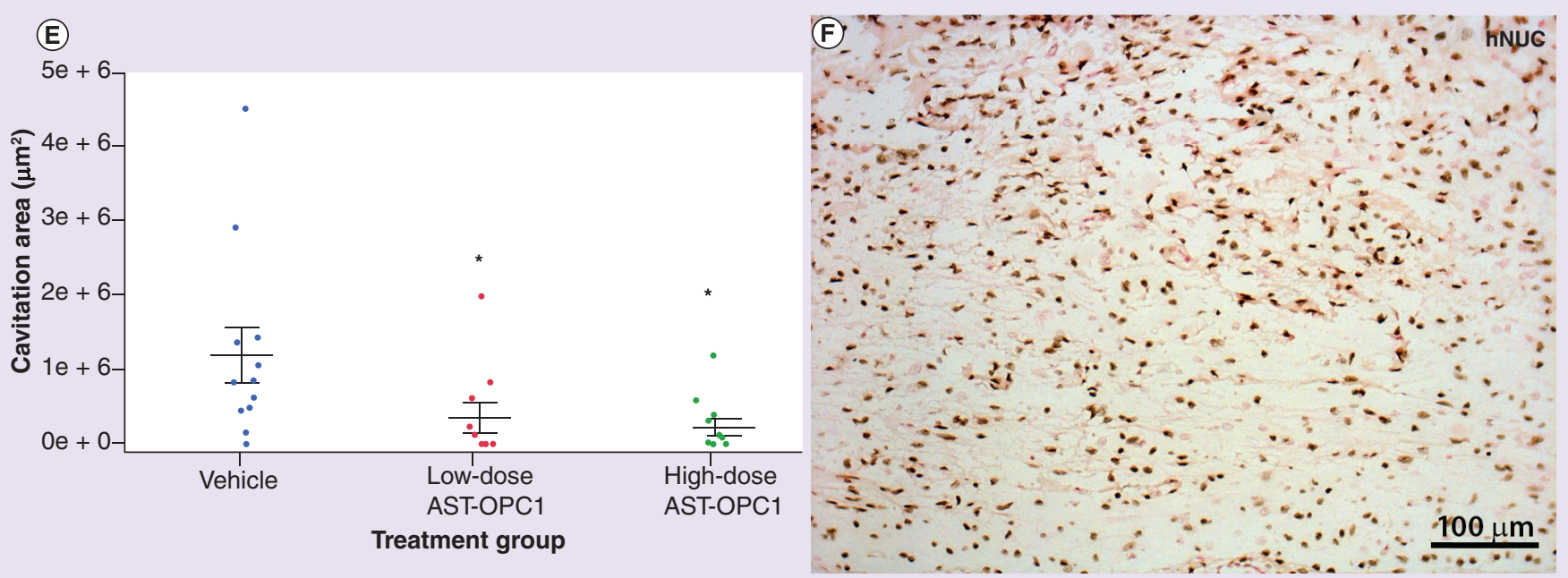

Figure 3. AST-OPC1 transplantation preserves host myelinated fibers and reduces cavity formation after thoracic spinal cord injury. (A \& D) Representative low and high magnification photomicrographs of the injury/graft site 9 months after thoracic spinal cord injury and injection of vehicle (A \& C) or transplantation of AST-OPC1 (B \& D). Sections were stained with Eriochrome cyanine to label myelin (blue) and counterstained with eosin. Black boxes in (A \& B) indicate the magnified regions shown in (C \& D). At 9 months post-contusion, vehicle-treated animals exhibited redirection of myelinated fibers around the lesion cavity (C), whereas cavitation was reduced in AST-OPC1-treated animals, and myelinated fibers were visible within the injury/graft site (D). 
Figure 3. AST-OPC1 transplantation preserves host myelinated fibers and reduces cavity formation after thoracic spinal cord injury (cont.). (E) Dot plot of cavitation area 9 months after thoracic spinal cord injury and vehicle or AST-OPC1 treatment. Asterisk denotes significance relative to vehicle treatment based on twotailed Student's t-test $(p<0.05)$. Horizontal lines denote mean plus standard error of the mean (SEM) for each treatment group (vehicle mean cavitation area $\pm \mathrm{SEM}=1.29 \pm 0.372 \mathrm{~mm}^{2}, \mathrm{n}=12$; low-dose AST-OPC1 mean cavitation area $\pm \mathrm{SEM}=0.378 \pm 0.201 \mathrm{~mm}^{2}, \mathrm{n}=10, \mathrm{p}=0.033$; high-dose AST-OPC1 mean cavitation area $\pm \mathrm{SEM}=0.244 \pm 0.111 \mathrm{~mm}^{2} \mathrm{n}=11, \mathrm{p}=0.0125$ ). (F) Representative photomicrograph of an adjacent tissue section from the same AST-OPC1-treated animal in (B \& D), showing positive labeling with a human Alu DNA repeat sequence probe by in situ hybridization (brown nuclear signal, eosin counterstain) within the same region exhibiting myelinated fibers (D). Magnification: $(A \& B)=25 x ;(C-F)=200 x$.

\section{Biodistribution of AST-OPC1}

We conducted a biodistribution study to examine the potential of AST-OPC1 to migrate and distribute within and outside the spinal cord following direct administration into the injured thoracic spinal cord. Two doses of cryopreserved AST-OPC1 $\left(2.4 \times 10^{5}\right.$ or $\left.2.4 \times 10^{6}\right)$ were administered into athymic nude rats 6-9 days after a 200 kdyne T9-10 spinal cord contusion injury and animals were maintained for 2, 14 and 180 days to examine biodistribution of the cells. These time points were chosen to reflect times before and after the expected restoration of the functional blood-spinal cord barrier, after which AST-OPC1 was considered unlikely to migrate out of the CNS [1]. Ten females were included at each of the three time points for each of the three treatment groups (HBSS vehicle control, $2.4 \times 10^{5}$ ASTOPC1 or $2.4 \times 10^{6}$ AST-OPC1). For the 180-day time point, an additional group of ten males was included which received the high dose of AST-OPC1. At 2, 14 and 180 days postadministration, five animals from each time point and treatment group were euthanized and had CNS and peripheral tissues collected including spinal cord (cervical cord/brainstem, lumbar/thoracic cord), brain (cerebellum, forebrain), blood, gonads, liver, heart, lung, kidney, spleen and small intestine. Samples were homogenized and assayed for the presence of human cells based on detection of hAlu DNA by qPCR. The lower limits of quantitation and detection for hAlu by qPCR in this assay was 1 pg human genomic DNA/ $\mu \mathrm{g}$ rat DNA and $100 \mathrm{fg}$ human genomic $\mathrm{DNA} / \mu \mathrm{g}$ rat DNA, respectively.

At all time points assayed, hAlu DNA was detected at less than the limit of quantification $(<1 \mathrm{pg}$ human gDNA $/ \mu$ g nude rat gDNA) in all rat peripheral tissues sampled, including blood, gonads, liver, heart, lung, kidney, spleen and small intestine. Out of the 280 peripheral tissue samples analyzed for hAlu DNA by qPCR, only nine $(3.2 \%)$ showed detectable but nonquantifiable levels of human DNA. This frequency was close to the incidence of false-positive samples (two of 120 or $1.7 \%$ ) observed in tissues from animals injected with HBSS. These data thus suggested that, if present, human cells were very rare in peripheral tissues.

At all time points, AST-OPC1 was found in the CNS, especially at the lesion site of the thoracic spi- nal cord (Figure 4A). In most cases, hAlu DNA was detected in the thoracic spinal cord at $>10,000 \mathrm{pg}$ human DNA per $\mu \mathrm{g}$ rat DNA ( $>1.0 \%$ human cells). The amount of human DNA in the thoracic spinal cord increased between 2 days and 180 days, although the difference in human DNA in the thoracic spinal cord did not appear to increase linearly with the dose of AST-OPC1.

In the cervical spinal cord, most animals had much reduced but measurable hAlu DNA levels ( $\geq 1-10,000$ pg human DNA/ $\mu$ g nude rat DNA). Two animals (1 at the 180-day time point and 1 at the 2 -day time point) that received $2.4 \times 10^{6}$ AST-OPC1 had high levels of hAlu DNA in this region $(\geq 10,000 \mathrm{pg}$ human DNA/ $\mu$ g nude rat DNA).

One rat in the 180-day termination group receiving $2.4 \times 10^{6}$ AST-OPC1 had high levels of hAlu DNA $(\geq 10,000 \mathrm{pg}$ human gDNA/ug nude rat gDNA) in the cerebellum. An additional three animals in the 180-day termination group had low levels of hAlu DNA in the cerebellum $(\geq 1-100 \mathrm{pg}$ human DNA/ $\mu \mathrm{g}$ nude rat DNA).

These PCR-based biodistribution results were confirmed by histological analysis of serial tissue sections of the thoracic spinal cord extending from caudal to the injury site, through the injury site, and rostral to the cerebellum from the remaining five animals per treatment group at each time point. The greatest concentration of human cells as identified by IHC for hNUC was at the injection site in area of the injury (Figure 4B). The concentration of parenchymal AST-OPC1 diminished with distance from the injury epicenter. AxioVision image analysis software (AxioVS $40 \mathrm{~V}$ 4.6.3.0, Carl Zeiss Imaging Solutions) was used to measure the maximum rostrocaudal length of spinal cord between hNUC-positive cells. The rostrocaudal extent of ASTOPC1 intraparenchymal distribution increased with time post-transplant, with distances of $15-17 \mathrm{~mm}$ observed between the most rostral and caudal migrating cells at the 180-day time point. AST-OPC1 migrated within the gray and white matter of the parenchyma and did not appear isolated by glial scarring or other anatomical barriers. Further analysis showed that, while greater numbers of AST-OPC1 migrated away from the injury epicenter in the animals that received the high 


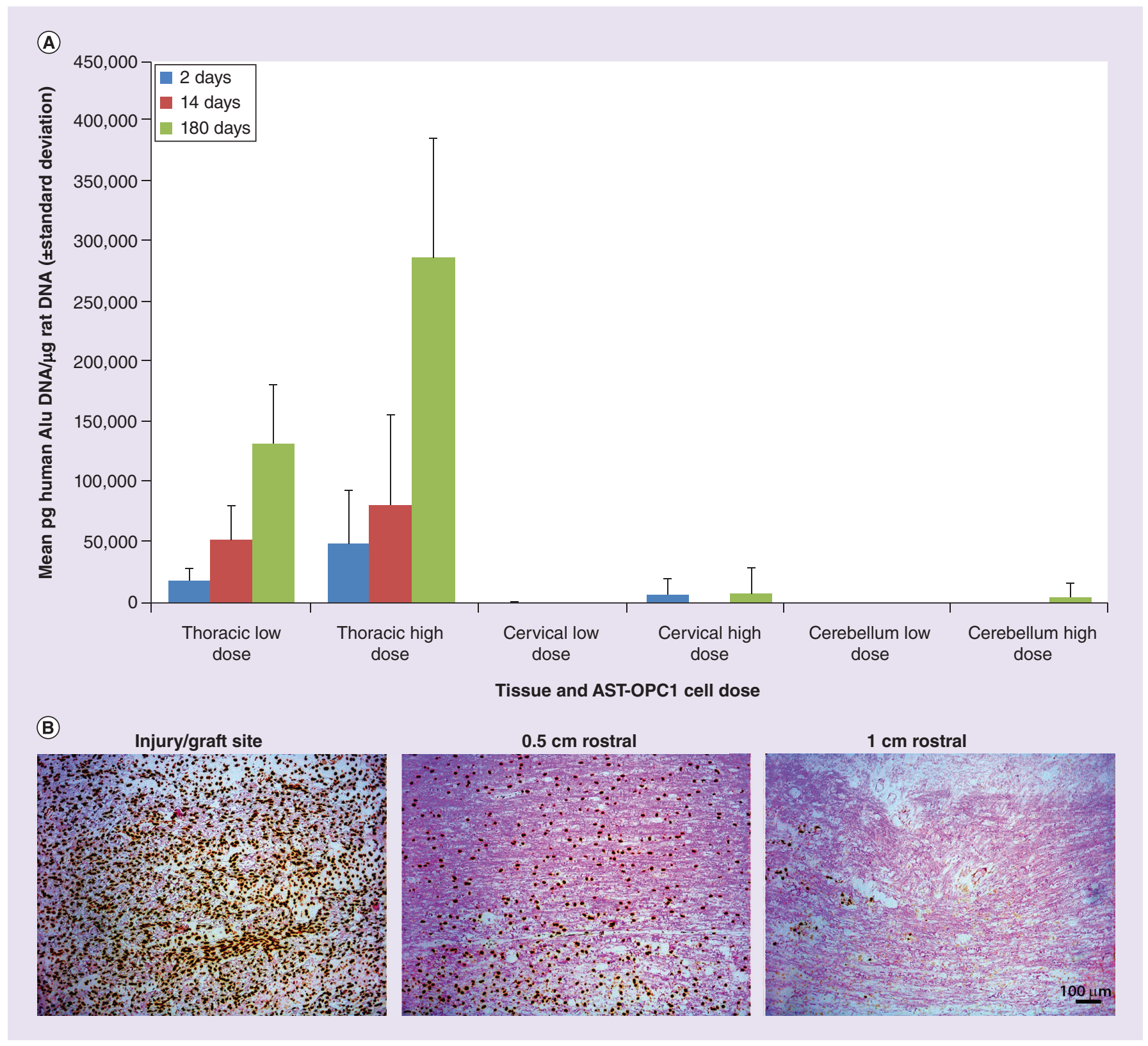

Figure 4. Quantification of human cell survival in the CNS in AST-OPC1-treated spinal cord injured rats. (A) Quantification of human DNA in rat spinal cord and cerebellum tissue as measured by quantitative human Alu sequence PCR. The graph depicts the mean pg of human DNA per microgram of rat DNA in the thoracic spinal cord, cervical spinal cord and in the cerebellum at 2, 14 and 180 days post-transplant of AST-OPC1 at the low $\left(2.4 \times 10^{5}\right.$ cells $)$ and high $\left(2.4 \times 10^{6}\right.$ cells $)$ dose. For each treatment group at each time point, $\mathrm{n}=5$. Error bars denote standard deviation. (B) Histological assessment of the presence of human cells at and rostral to the injury site at 180 days post-administration as determined by immunohistochemistry using antibodies against human nuclear antigen (hNUC). The human cell nuclei appear brown in pink eosin-counterstained tissue. Magnification $=100 \times$.

dose of cells, the extent of migration was independent of the cell dose administered in this study.

\section{Toxicology assessments of AST-OPC1}

Three toxicology studies were conducted in accordance with Good Laboratory Practices (GLP) to investigate the potential toxicity of AST-OPC1 in the injured spi nal cord. These studies specifically addressed potential toxicity issues of AST-OPC1 related to its delivery to the thoracic spinal cord, impact on organ function, induction of allodynia and tumorigenicity. For the toxicology studies, a rat spinal cord contusion injury was utilized to mimic as closely as possible the conditions that would be encountered in patients with nonlacerating spinal cord crush injuries. In each study, rats were given a moderate 200 kdyne contusion injury 
and transplanted with either cryopreserved AST-OPC1 or HBSS vehicle. For transplantation, cryopreserved, thawed and prepared AST-OPC1 was injected approximately 6-9 days postinjury into the lesion site of these animals using the same delivery methodology intended for use in the proposed clinical trial. The three toxicology studies examined contused male and female rats, of which a total of 299 and 285 were injected with vehicle and AST-OPC1, respectively. AST-OPC1 doses of $2.4 \times 10^{5}$ and $2.4 \times 10^{6}$ cells were assessed.

Vehicle and AST-OPC1 transplanted rats were examined at 2, 6, 9 and 12 months postinjury for any negative impact on systemic organ function as measured by testing blood and urine analytes. Supplementary Table 3 describes the metabolic and hematologic parameters examined to assess the potential systemic toxicity of AST-OPC1. The collective data from these studies suggested that AST-OPC1 did not induce any significant alterations in hematology, coagulation, urinalysis or clinical chemistry parameters compared with injured vehicle control animals. Some elevation in individual parameters, especially in liver enzymes and urea nitrogen were observed occasionally in animals receiving either AST-OPC1 or vehicle, likely due to the spinal cord injury itself or to the prolonged use of immunosuppression. There were no statistically significant differences in the mortality, body weights or clinical observations including behavioral activity, excretion, external appearance or skin condition between the AST-OPC1 and vehicle-treated contused rats. Mortality was relatively high across treatment groups $($ AST-OPC $1=47 \%$; vehicle $=49 \%$ ), and was observed throughout the 'in-life' phase of the studies. A common cause of death in all groups was septicemia/inflammation, not related to AST-OPC1 but likely a reflection of the immunocompromised state of the animals and their spinal cord injury. Many deaths were also related to urogenital dysfunction, inflammation, obstruction and/or calculi. The occurrence or frequency of these urogenital events was not considered related to AST-OPC1, as they were observed with similar frequency in the vehicle group.

Many individuals with SCI report musculoskeletal, neuropathic and visceral pain. To investigate this, all

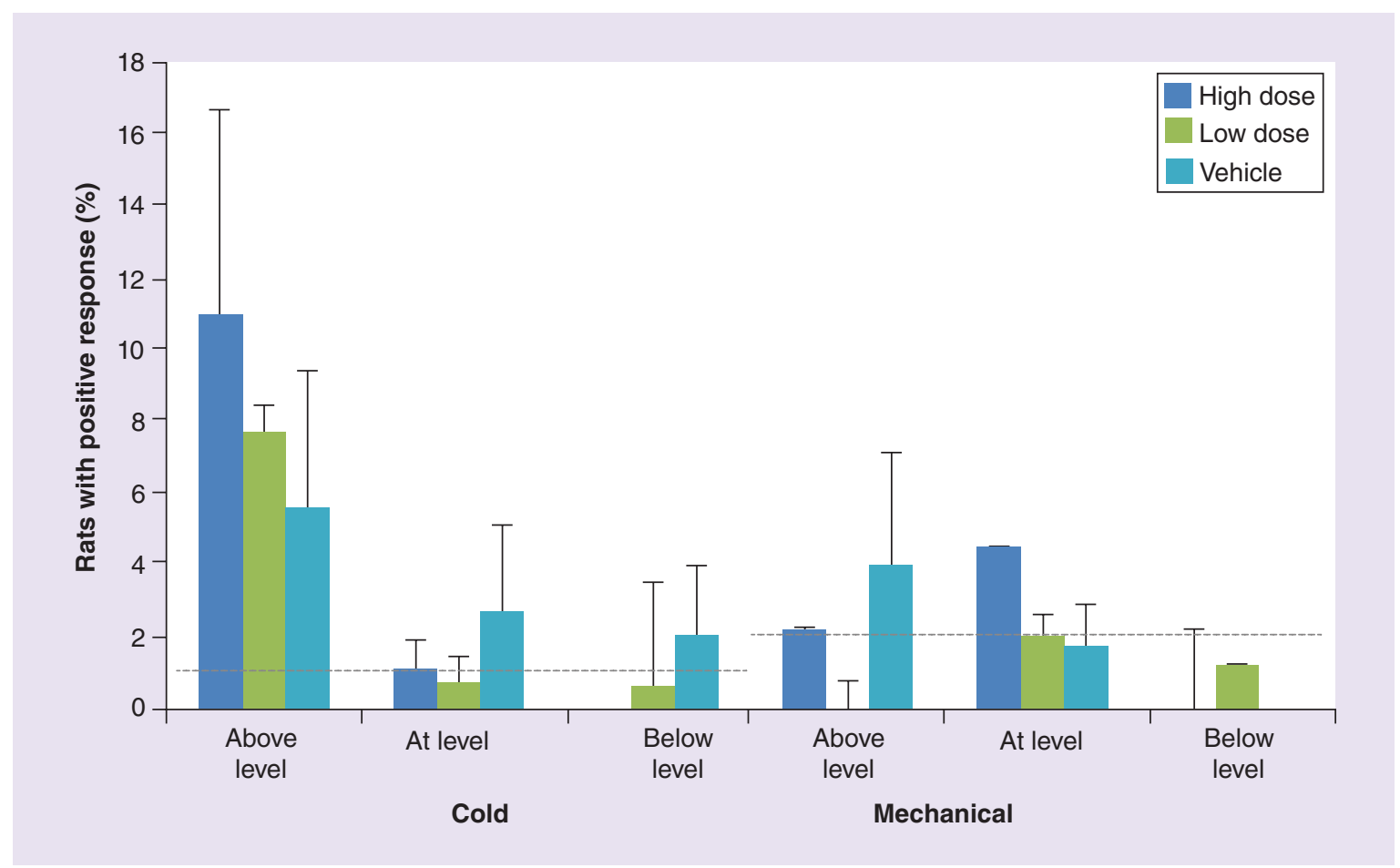

Figure 5. Measurement of allodynia 9 months after administration of AST-OPC1 in spinal cord injured rats. At 9 months post-transplantation, rats receiving vehicle or the low or high dose of AST-OPC1 were assessed for hypersensitivity to normally non-noxious mechanical (blunt probe) or cold (point application of acetone) stimuli. Up to ten randomly selected animals per sex per treatment group were examined for the development of allodynia. The percentage of rats with positive responses to a stimulus is shown, with error bars showing standard error of the mean (high-dose AST-OPC1, $n=2$ male/8 female; low-dose AST-OPC1, $n=9$ male/8 female; vehicle, $\mathrm{n}=4$ male/ 6 female). The percentage of positive responses was compared across treatment groups using the Kruskal-Wallis test, a nonparametric version of a one-way ANOVA. No significant differences were observed. Horizontal dotted gray lines indicate the mean values of uninjured (laminectomy only) vehicle-treated animals 9 months postinjection for each stimulus type (cold stimulus $=1 \%$, mechanical stimulus $=2 \%$ ). 
animals that received SCI were monitored for autophagia, and other general behavioral indicators of pain. Across the three toxicology studies, only seven of 584 injured rats (three vehicle and four AST-OPC1) showed signs of autophagia.

Additional behavioral tests were performed to assess if AST-OPC1 impacted the frequency of allodynia in rats with SCI. At approximately 3, 6 and 9 months post-transplantation, animals in the largest toxicology study were evaluated for allodynia, hypersensitivity in response to normally non-noxious mechanical (blunt probe) or cold (point application of acetone) stimuli. To assure that the mechanical probe and cold (acetone) stimuli were nonaversive, the methods were initially used on the dorsal skin surface of 18 random male and 20 random female healthy rats from the study prior to laminectomy to establish baseline responses. Each animal tested was observed for supraspinal responses to the stimuli according to the following parameters: three anatomical levels of assessment (at, above and below the level of injury), two modalities (application of mechanical stimulation or cold stimulation) and two assay sites (dorsal skin surface of the trunk and glabrous tissue of the paws). At 9 months postadministration, animals treated with AST-OPC1 did not show significant changes in their response profile, relative to vehicle controls, for either modality or at any anatomical sites, and did not display signs of allodynia (Figure 5). Data are expressed as percent positive responses.

\section{Tumorigenicity assessment of AST-OPC1}

In the toxicology studies described above, macroscopic and microscopic examinations were performed by an independent veterinary pathologist who was blinded to the animals' treatment groups to ascertain whether AST-OPC1 administration resulted in any particular pathology either within or outside the CNS. Such analysis also included assessments for teratoma or ectopic tissue formation. For this analysis, teratomas were defined as expansile proliferations or masses which appeared to have arisen from at least two different embryonic germ layers (endodermal, mesodermal and/or ectodermal). Ectopic tissue was defined as tissue not normally occurring in the tissue or organ examined. For these analyses, over 50 tissues were examined from each animal including the longitudinal extent of the spinal cord and five levels of the brain. There were no macroscopic or microscopic pathologic findings outside the spinal cord that were associated with AST-OPC1 in either male or female rats from the 2-, 6- or 9-month termination groups.

The entire length of the thoracic and cervical spinal cords and five levels of the brain (medulla/pons, cer- ebellum, midbrain, forebrain and olfactory bulbs) were examined for teratomas and ectopic tissue. Teratomas were not observed in the spinal cord or brain in any of the 252 animals examined 2, 6 or 9 months postadministration. In agreement with this, cells within the injury/graft site exhibited very low positivity for the proliferation marker, Ki-67, at 9 months post-administration (Supplementary Figure 1). Furthermore, ISH labeling with an hAlu probe indicated robust graft survival in the thoracic spinal cord of 239 out of the 252 assessed animals (95\%) examined 2, 6 or 9 months post-administration (Supplementary Figure 1).

In six of the 252 animals injected with AST-OPC1, microscopic 'cystic-like epithelial structures' were observed. Out of the six observed instances of cyst formation, two were observed in animals that received a dose of $2.4 \times 10^{5}$ AST-OPC1 and four were observed in animals that received a dose of $2.4 \times 10^{6}$ AST-OPC1. These cystic structures were confined to the lesion site, ranged in size from 34 to $980 \mu \mathrm{m}$ in diameter and were smaller than typical parenchymal cavitation seen in vehicle controls. In three of these six animals, two to three separate cystic structures were observed in close proximity to one another in the histological sections and may have represented lobules of a single structure. A thoracic spinal cord tissue section containing one of these cystic structures is shown in Figure 6. Cystic structures were lined with cells exhibiting epithelial morphology (Figure 6B), were of human origin (Figure 6C) and were not highly proliferative given that very few cells were positive for the proliferative marker, Ki-67 (Figure 6D). Cystic structures were never observed in healthy tissue or in the cervical spinal cord or brain of any animal in the study. There were no apparent clinical symptoms in the animals in which cystic structures were observed.

\section{Additional teratoma assessments of AST-OPC1}

The potential for teratoma formation by AST-OPC1 was also tested in the spinal cord of $\mathrm{CB}-17 / \mathrm{IcrCrl}-$

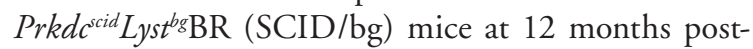
administration for two different lots of AST-OPC1. In these studies, $2 \times 10^{6}$ AST-OPC1 were administered to the intact, uninjured T10 thoracic spinal cord of the immunodeficient mice. A positive control group received undifferentiated hESCs, and a negative control group received HBSS vehicle alone. Additional treatment groups received a total of $2 \times 10^{6}$ cells containing AST-OPC1 spiked with $1,5,10$ or $50 \%$ undifferentiated hESCs. Treatment groups comprised an equal number of males and females and were followed for 12 months to assess tissues for teratoma formation. Animals euthanized prior to 12 months of follow-up were also examined at termination for teratoma for- 


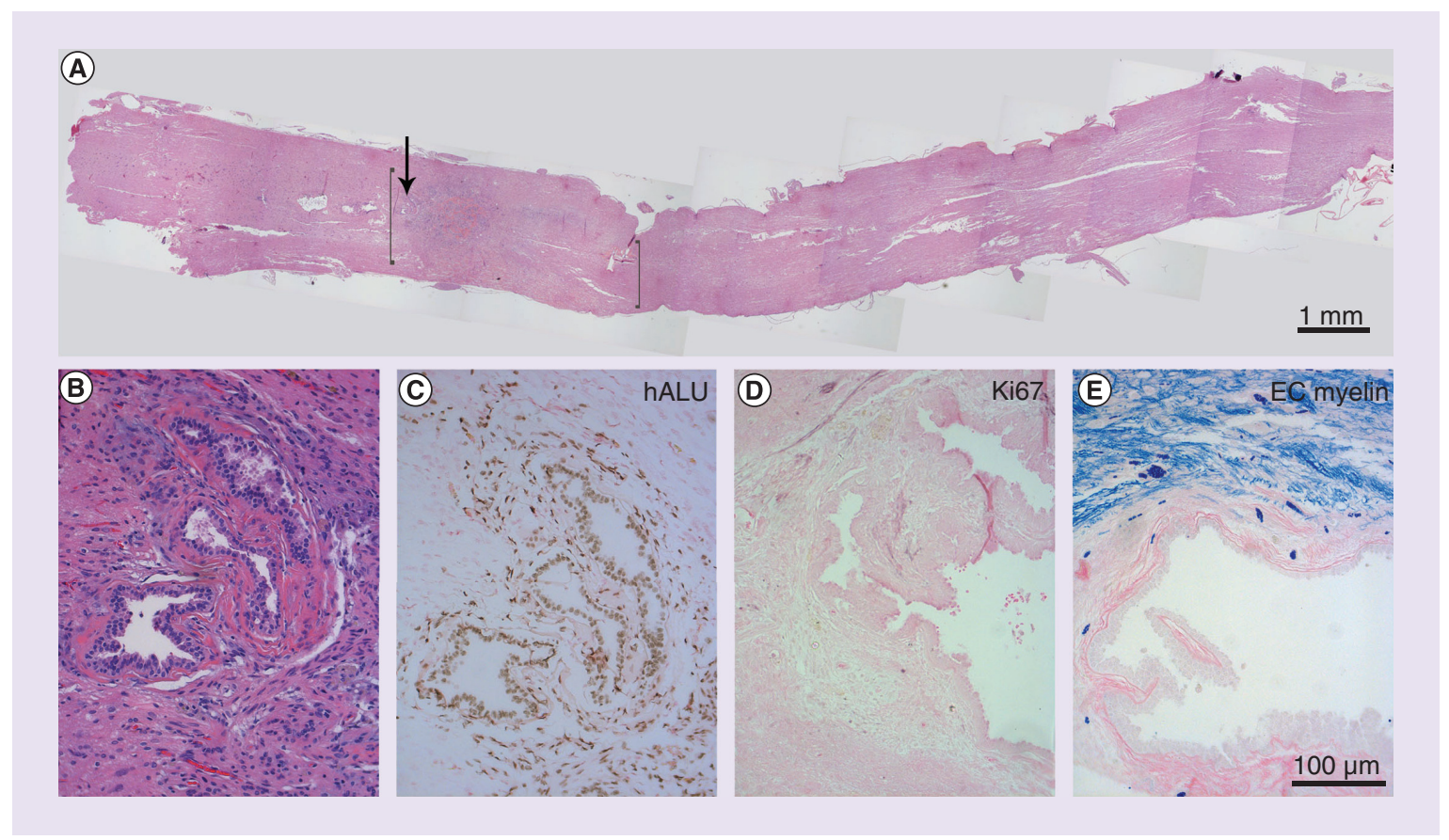

Figure 6. Presence of a cystic-like epithelial structure of human origin within the injury/graft site 9 months after contusion injury and AST-OPC1 treatment. (A \& B) Low and high magnification hematoxylin and eosin staining of thoracic spinal cord from an animal treated with AST-OPC1 in which a cystic structure was observed 9 months post-treatment. In a photographic montage of the contused spinal cord (A), brackets indicate the approximate boundaries of the highest graft density, and the cystic structure is indicated with a black arrow. To the left of the cystic structure, just caudal to the indicated graft boundary, residual cavitation is apparent. (C) The cystic structure was comprised of human cells based on positive labeling with a human Alu DNA repeat sequence probe (brown nuclear signal, eosin counterstain). (D) Few Ki-67-positive cells were detectable by immunohistochemistry within the injury/graft site or within the cystic structure. (E) Myelinated fibers labeled with Eriochrome cyanine (blue) were detectable within the injury/graft site and immediately adjacent to the cystic structure. Magnification: $(B-E)=200 x$.

mation. ISH with hAlu probes was performed for all recovered spinal cords and confirmed that human AST-OPC1 persisted in $>97 \%$ of animals.

No teratomas were observed in mice that were injected in the uninjured spinal cord with vehicle alone. Nine of $20(45 \%)$ of these vehicle control animals survived to the 12 -month termination point. By contrast, teratomas were observed in the majority of animals injected with $100 \%$ undifferentiated hESCs. In these animals, 20 of the $30(67 \%)$ mice injected with $2 \times 10^{6}$ undifferentiated hESCs developed teratomas in the spinal cord (Figure 7). Only six of the 30 $(20 \%)$ animals survived to the 12 -month termination point. None of the 128 mice injected with $2 \times 10^{6}$ AST-OPC1 and examined histologically showed any evidence of teratomas and 51\% survived throughout the 12-month in-life phase. In addition, AST-OPC1 spiked with $50 \%$ undifferentiated hESCs produced teratomas in approximately $70 \%$ of mice. With lower numbers of undifferentiated hESCs, the frequency of teratoma formation decreased. Only 13\% (four of 31) mice receiving AST-OPC1 spiked with 10\% hESCs produced teratomas, whereas AST-OPC1 spiked with
5 and $1 \%$ undifferentiated hESC did not lead to teratoma formation ( 0 of 12 and 0 of 36, respectively, Figure 7). Summaries of the design and key findings of this tumorigenicity study, as well as the toxicology studies and biodistribution study are presented in Table 1.

\section{Discussion}

The damage that results from traumatic SCI is complex and will likely require multiple mechanisms for repair. Use of single agents has thus far failed to demonstrate efficacy in spite of a large number of human clinical trials. To address this, investigators have explored the use of combinatorial therapies in animal models of SCI, while others have explored administration of multifunctional progenitor cells, and preclinical results have been encouraging. Combined use of such treatments as neurotransmitter agonists, neurotrophinsecreting cells, antigrowth inhibitors and exercise have been shown to increase axonal outgrowth and synaptic plasticity, suppress muscle atrophy and improve functional recovery in a synergistic manner [25,26]. Several groups have shown that transplantation of neural/glial 


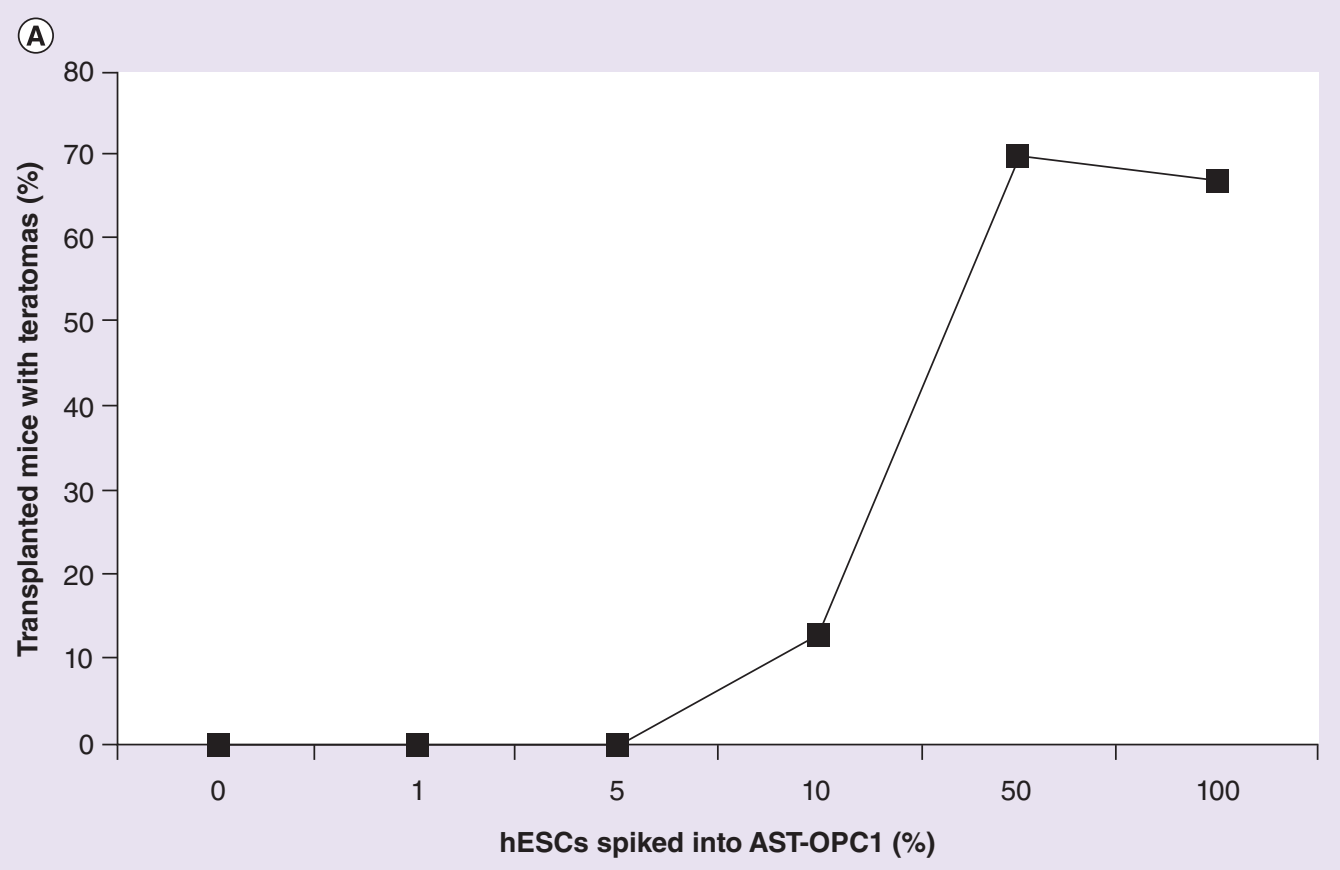

(B)

\begin{tabular}{|l|c|c|c|}
\hline \multicolumn{1}{|c|}{ Spiking group } & $\begin{array}{c}\text { Total cells } \\
\text { injected }(\mathbf{n})\end{array}$ & $\begin{array}{c}\text { Total hESCs } \\
\text { injected }(\mathbf{n})\end{array}$ & $\begin{array}{c}\text { Mice with teratomas }(\mathbf{n}) / \\
\text { total mice examined }(\mathbf{n})\end{array}$ \\
\hline $100 \% \mathrm{hESCs}$ & $2 \times 10^{6}$ & $2 \times 10^{6}$ & $20 / 30$ \\
\hline $50 \% \mathrm{H} 1 \mathrm{hESCs}+50 \% \mathrm{AST}-O P C 1$ & $2 \times 10^{6}$ & $1 \times 10^{6}$ & $21 / 30$ \\
\hline $10 \% \mathrm{H} 1 \mathrm{hESCs}+90 \%$ AST-OPC1 & $2 \times 10^{6}$ & $2 \times 10^{5}$ & $4 / 31$ \\
\hline $5 \% \mathrm{H} 1 \mathrm{hESCs}+95 \%$ AST-OPC1 & $2 \times 10^{6}$ & $1 \times 10^{5}$ & $0 / 12$ \\
\hline $1 \% \mathrm{H} 1 \mathrm{hESCs}+99 \%$ AST-OPC1 & $2 \times 10^{6}$ & $2 \times 10^{4}$ & $0 / 36$ \\
\hline $100 \%$ AST-OPC1 & $2 \times 10^{6}$ & 0 & $0 / 128$ \\
\hline Vehicle & 0 & 0 & $0 / 30$ \\
\hline
\end{tabular}

Figure 7. Frequency of teratoma formation in mice treated with AST-OPC1 or AST-OPC1 spiked with increasing levels of $\mathrm{H} 1$ undifferentiated human embryonic stem cells. (A) Frequency of teratoma formation is plotted against the percent of undifferentiated hESCs spiked into AST-OPC1. (B) For each treatment group, number of cells administered and sample size are shown. hESC: Human embryonic stem cell.

progenitor cells in SCI models can augment multiple mechanisms of repair, including trophic support, cavity reduction, axon outgrowth, synaptic plasticity, remyelination and immunomodulation [6,12,27-29]. Because neural/glial progenitor cells typically possess the capacity for migration, proliferation and multipotent differentiation, they have the added potential to impact large areas of injured tissue and replace endogenous populations of neurons, astrocytes and oligodendrocytes [30-32]. Given these promising preclinical results, use of neural/glial progenitors may prove more effective than other approaches to stimulate repair after SCI.

AST-OPC1 is an oligodendrocyte progenitor cell therapy product derived from hESCs and developed as a treatment for SCI. Based on molecular profiling of more than 75 manufactured AST-OPC1 lots, the AST-OPC1 differentiation process produces a cellular phenotype consistent with early-stage oligodendrocyte progenitors [9], with minimal levels of mature neurons 


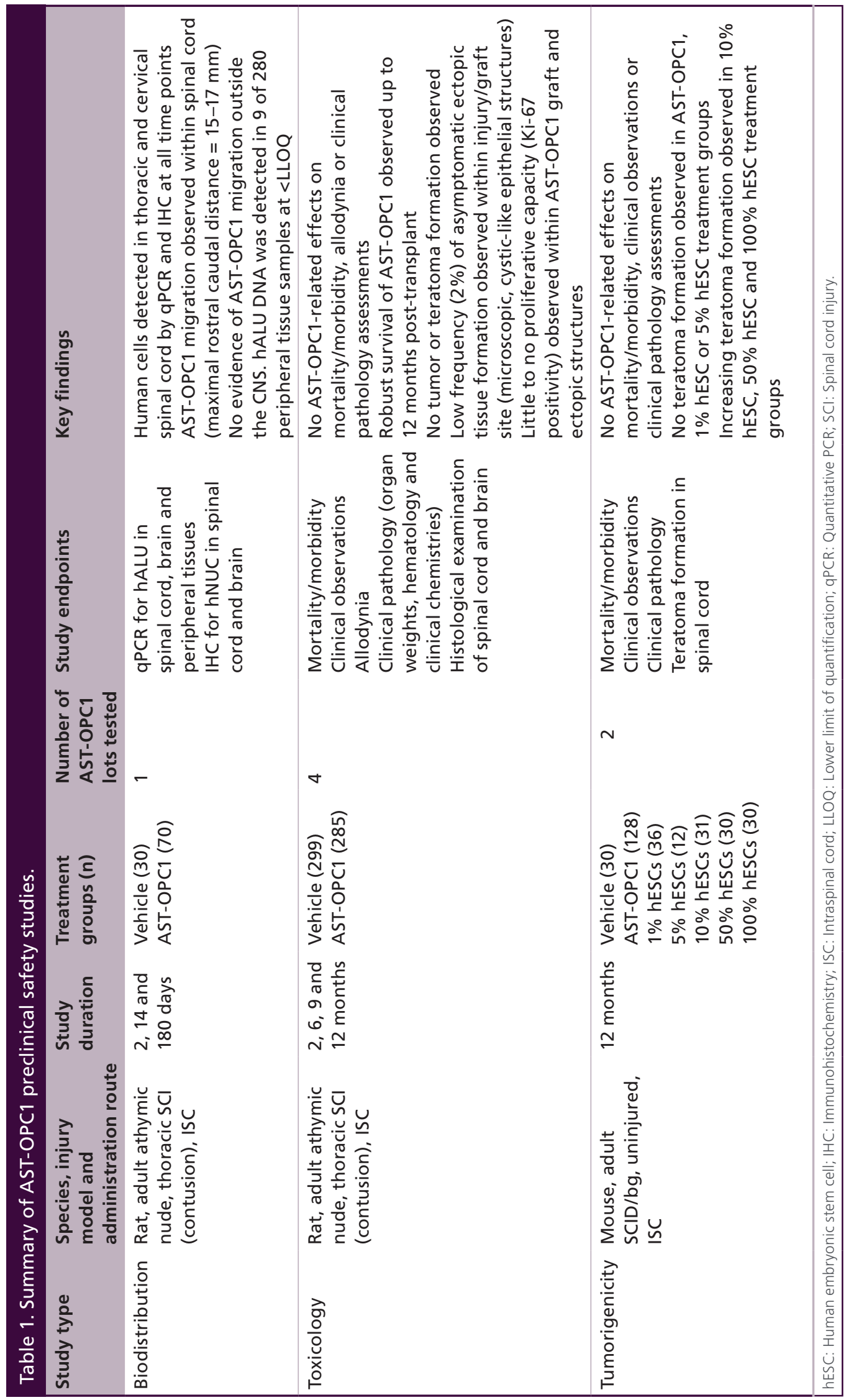


or glia. This contrasts with alternative hESC-based OPC protocols that use small molecules, additional growth factors or longer differentiation processes to stimulate further OPC maturation [9,33-34].

Prior studies and results presented here indicate that AST-OPC1, when administered during the subacute phase of SCI, can act via multiple repair pathways that are relevant to SCI, including trophic factor signaling, cavity reduction and stimulation of axon outgrowth and myelination $[7,10,35]$. Furthermore, recent studies have shown that immature OPCs, but not mature oligodendrocytes, promote functional recovery when administered subacutely post-SCI and the lack of benefit with mature oligodendrocytes may be partly due to their expression of surface proteins that are inhibitory to axonal outgrowth [36]. Finally, it is likely that AST-OPCl's capacity for robust engraftment and subsequent migration is a result of its relatively immature state [9], thus allowing for preservation and repair of tissue beyond the initial injury/graft site. Collectively, these phenotypic and functional data support the continued development of AST-OPC1 as a subacute treatment for SCI, while also identifying key safety concerns prior to clinical testing.

The majority of safety testing was conducted in nude rats subjected to thoracic SCI, providing a well-established model of the target clinical population [37-39], and additional tumorigenicity studies were conducted in uninjured SCID/bg mice. Importantly, these rodent models enabled testing of AST-OPC1 in a large number of subjects and in an immunocompromised environment that was permissive to human cell survival. both of these attributes facilitated assessment of key safety concerns associated with AST-OPC1 administration, including the resultant biodistribution, toxicity and tumorigenic potential of the transplanted cells. As one limitation of these preclinical studies, rats subjected to an incomplete midline contusion injury were used to support clinical testing in sensorimotor complete thoracic SCI. Although it is critically important for preclinical studies to model the target clinical population as accurately as possible, use of an incomplete injury model in this case was instead based on the need to maximize animal survival and allow for assessment of long-term cell persistence.

To address the migratory capacity of AST-OPC1, we performed biodistribution studies in injured nude rats and asked whether AST-OPC1 migration was restricted to the spinal cord or also extended into the brain or peripheral tissues. That such long distance migration poses a potential safety concern has been demonstrated clinically, with recent reports of other cell therapy products, including fetal-derived tissue grafts, migrating to unintended locations and even causing remote tumors [40,41]. In the case of ASTOPC1, it was particularly important to use injured rats given the potential for increased exchange between the spinal cord parenchyma, CSF and circulatory system during the subacute phase of SCI [1-3]. To detect human cells, we performed PCR-based quantification of human microsatellite DNA, providing the highest level of sensitivity currently available $\left(1\right.$ human cell $/ 1 \times 10^{6}$ rat cells), coupled with histological labeling of human cells by ISH. Based on these approaches, AST-OPC1 exhibited robust persistence and limited migration within the thoracic and cervical spinal cord, and was either extremely rare, registering below quantifiable levels of human DNA in a few animals, or altogether absent from peripheral tissues. In addition, detection of human DNA in the cerebellum of four animals may have reflected human DNA contamination during sample processing, as very few human cells were detected in the animal's adjacent cervical spinal cord, a site more proximal to the injection site and false-positive values were also detected in a small number of vehicletreated animals. Furthermore, it was unclear whether the observed increase in human DNA in the thoracic spinal cord from 2 to 180 days was indicative of human cell proliferation or was due to loss of human cells during tissue collection at the earlier time points due to tissue fragility. Overall, these findings support the general safety of administering AST-OPC1 into the spinal cord parenchyma subacutely post-SCI and indicate minimal risk of the transplanted cells reaching unintended locations.

Perhaps the greatest theoretical safety concern associated with hESC-derived and progenitor cell therapies is their potential for tumor formation resulting either from incomplete removal of the starting hESC population or overactive proliferation by the progenitors themselves. We characterized the tumorigenic potential of AST-OPC1 in both injured adult nude rats and uninjured adult SCID/bg mice, assessing large cohorts of male and female animals for up to 12 months. Within both of these systems, we observed robust survival of AST-OPC1 in almost all assessed animals (i.e., 95\% of assessed rats) and detected no signs of tumor or teratoma formation. These findings likely reflect the minimal presence of Oct4-positive cells in AST-OPC1. While flow cytometry indicated $<0.2 \%$ Oct 4 positivity in AST-OPC1 (lower limit of detection), a high-content imaging assay under development at Asterias indicates that the actual level of Oct4-positive cells in AST-OPC1 is much lower than $0.2 \%$ (data not shown). Based on our hESC spiking study in SCID/bg mice, hESC levels as high as 5\% (corresponding to 50,000 undifferentiated hESCs) 
did not result in teratoma formation. The results of this hESC spiking study contrasts with prior reports, in which far fewer hESCs implanted within different anatomical locations of immunodeficient mice were sufficient to induce teratoma formation [42-44]. Our findings likely reflect a decrease in tumorigenic potential of hESCs following the AST-OPC1 harvest and dose preparation method (including enzymatic dissociation to single cells), as well as their administration into the spinal cord, both of which model the intended clinical use of AST-OPC1.

In addition to the lack of tumorigenic potential observed for AST-OPC1, administration of these cells into the injured spinal cord was not associated with any adverse clinical observations, systemic or local toxicities up to 12 months post-transplant. However, AST-OPC1 did exhibit a low frequency of ectopic tissue formation, in the form of cystic-like epithelial structures present within the injury/graft site of six of 252 assessed rats. In all cases, cystic structures were rare (1-3 cysts/rat), microscopic in size $(980 \mu \mathrm{m}$ maximum diameter), exhibited little to no proliferative capacity and were clinically asymptomatic.

It remains unclear as to whether AST-OPC1's low capacity for ectopic tissue formation was intrinsic to the cells themselves or also resulted from regenerative signaling in the host tissue postinjury, as it is known that ectopic tissue, in the form of fibrosis, gliosis and dystrophic calcification, is observed in clinical SCI [3]. While these findings identify an important safety consideration for AST-OPC1, its small capacity for nonproliferative ectopic tissue formation is in stark contrast to the more aggressive tumorigenic profiles reported with other cell types, including hESC-derived neural cells [45-47], fetal-derived neural cells [40], adult stem cells [41] and mesenchymal stromal cells [48]. Going forward, it will be important to understand the origin of these rare ectopic tissues and to modify the production process of AST-OPC1 to reduce their frequency.

In addition, it will be important for future preclinical studies to explore in greater detail the potential mechanisms by which AST-OPC1 can improve outcome following SCI. As a key component of this understanding, it will be necessary to characterize the kinetics and relative proportion of transplanted cells that become mature oligodendrocytes or differentiate into other cell types. In addition, future studies must determine the extent to which repair mechanisms such as trophic support, extracellular matrix remodeling, cavity reduction and remyelination drive AST-OPC1's capacity to improve functional recovery, as this will help guide future improvements to the AST-OPC1 production process.
These collective preclinical safety studies provided valuable information that directly contributed to the design of the first-in-human study. For instance, the highest feasible dose that was injected into the injured rat, $2.4 \times 10^{6}$ cells, provided rationale for the dose of $2 \times 10^{6}$ cells as the starting dose in humans. A single injection site at the lesion was selected for initial clinical testing given the observed migration of AST-OPC1 in the preclinical biodistribution study. In addition, since cell migration was not detected outside of the CNS, safety assessments in the clinical trial were primarily focused on the spinal cord and brain. The toxicology studies also informed the initial clinical dosing window, as delivery of AST-OPC1 in the subacute period postinjury was found to promote long-term engraftment without significant toxicity. However, one noteworthy difference between the rodent toxicology studies and the subsequent thoracic SCI clinical trial was the volume used for AST-OPC1 administration. For the rodent studies, large volumes $(24 \mu \mathrm{l} / \mathrm{rat}, 10 \mu \mathrm{l} /$ mouse $)$ were used to administer maximal doses of AST-OPC1 and thereby increase the likelihood of detecting any side effects associated with AST-OPC1, particularly with respect to its capacity to form tumors or ectopic tissue. In contrast, the highest planned dose for clinical testing $\left(2 \times 10^{7}\right.$ cells/patient) will require an administration volume that is approximately 12 -fold lower than the volume administered to rats. As such, it will be important for future preclinical studies to identify the optimal cell administration parameters for SCI, including injection volume and injection rate, as this will help guide and refine the clinical use of AST-OPC1.

\section{Conclusion}

Based on the safety data presented here and prior efficacy studies of AST-OPC1, the risk/benefit assessment of this cell therapy product was considered favorable, and AST-OPC1 was cleared by the FDA for Phase I clinical testing in patients with thoracic SCI. The trial includes plans to collect extensive patient safety data for up to 15 years, and therefore is expected to provide valuable information regarding the clinical suitability of AST$\mathrm{OPC} 1$ and related cell therapy products. Following the acquisition of the AST-OPC1 program by Asterias, an IND amendment was filed with the FDA that cleared AST-OPC1 for clinical testing in patients with complete cervical SCI. A manuscript detailing the results of the preclinical safety and efficacy studies that supported advancement into cervical SCI is in preparation.

\section{Future perspective}

This manuscript describes the preclinical safety studies that were required to enable the first hESC-based therapy (AST-OPC1) to be tested clinically in patients 
with complete thoracic spinal cord injuries. The results from these preclinical studies provided fundamental information about the cell therapy that guided the choice of clinical injection parameters and specific follow-up assessments that were important to establish the overall behavior and safety of AST-OPC1. It is information from these types of preclinical investigations that will also enable expansion of the clinical trials into patients with cervical spinal cord injury and ultimately other CNS diseases. The importance of preclinical studies will remain high for all stem cellbased therapies with increasing need for representative and predictive models of human disease.

\section{Supplementary data}

To view the supplementary data that accompany this paper please visit the journal website at: www.futuremedicine.com/ doi/full/10.2217/rme.15.57

\section{Acknowledgements}

The authors would like to thank the following individuals for their contributions: Elham Yeganeh and manufacturing colleagues for CGMP production of AST-OPC1, Sandra Powell for performing flow cytometry experiments, Scott Thies for performing ICC experiments, Karen Delavan-Boorsma for performing the rat $\mathrm{SCl}$ model, Samantha Edell for animal husbandry support; Ross Okamura for Shiverer mouse breeding, Yelena Polonskaya for performing histological analyses, Amanda Conta for performing cavitation measurements, Ra- chel Tapp for directing toxicology studies at MPI Research and Casey Case for help with the manuscript.

Financial \& competing interests disclosure

All work described in this study was funded by Geron Corporation or Asterias Biotherapeutics, Inc. J Lebkowski, E Wirth and N Manley are employed by Asterias Biotherapeutics, Inc. In USA and internationally, patents have been filed and issued for the AST-OPC1 product. The authors have no other relevant affiliations or financial involvement with any organization or entity with a financial interest in or financial conflict with the subject matter or materials discussed in the manuscript apart from those disclosed.

No writing assistance was utilized in the production of this manuscript.

\section{Ethical conduct of research}

The authors state that they have obtained appropriate institutional review board approval or have followed the principles outlined in the Declaration of Helsinki for all human or animal experimental investigations. In addition, for investigations involving human subjects, informed consent has been obtained from the participants involved.

\section{Open access}

This work is licensed under the Creative Commons Attribution-NonCommercial 4.0 Unported License. To view a copy of this license, visit http://creativecommons.org/licenses/bync-nd/4.0/

\section{Executive summary}

\section{Description of AST-OPC1}

- AST-OPC1 is a cell therapy product that is produced through differentiation of human embryonic stem cells into early oligodendrocyte progenitor cells (OPCs) that are positive for Nestin, NG2, Olig1 and PDGFR- $\alpha$.

- Based on flow cytometry- and immunocytochemistry-based analyses of over 75 manufactured lots, AST-OPC1 contained minimal levels of other unwanted cell types, such as human embryonic stem cells and endoderm/ mesoderm lineage cells and also lacked mature neurons and astrocytes.

\section{Biological activities of AST-OPC1}

- AST-OPC1 conditioned medium contained multiple secreted factors relevant to brain and spinal cord repair, including clusterin, MCP-1, TIMP-1 and 2 and ApoE, and promoted neurite outgrowth in cultured primary cortical neurons and myelination in shiverer mice.

\section{Biodistribution of AST-OPC1}

- AST-OPC1 cells administered into the injured thoracic spinal cord of nude rats exhibited robust survival for up to 1 year post-transplant and limited migration within the spinal cord (approximately $1 \mathrm{~cm}$ rostral and caudal of the injury/graft site), and little to no cells were detected in the brain and peripheral organs up to 9 months post-transplant.

\section{Toxicology \& tumorigenicity assessments of AST-OPC1}

- Administration of AST-OPC1 into the rodent spinal cord did not cause adverse clinical observations, systemic toxicities or allodynia, and transplanted cells showed no evidence of tumor or teratoma formation.

- A low frequency (2\%) of treated animals exhibited one to three microscopic cystic-like epithelial structures of human origin within the injury/graft site that were nonproliferative and clinically asymptomatic.

\section{Conclusion}

- A clinical trial of AST-OPC1 administration in patients with sensorimotor complete spinal cord injury was initiated, and it is expected that ongoing collection of the clinical safety data up to 15 years post-treatment will provide critical information with regard to the clinical use of AST-OPC1 and related cell therapies. 


\section{References}

Papers of special note have been highlighted as:

- of interest; $\bullet \bullet$ of considerable interest

1 Kakulas BA. The applied neuropathology of human spinal cord injury. Spinal Cord 37(2), 79-88 (1999).

2 Anderson DK, Hall ED. Pathophysiology of spinal cord trauma. Ann. Emerg. Med. 22(6), 987-992 (1993).

3 Norenberg MD, Smith J, Marcillo A. The pathology of human spinal cord injury: defining the problems. J. Neurotrauma 21(4), 429-440 (2004)

4 Anderson KD, Friden J, Lieber RL. Acceptable benefits and risks associated with surgically improving arm function in individuals living with cervical spinal cord injury. Spinal Cord 47(4), 334-338 (2009).

5 Almad A, Sahinkaya FR, Mctigue DM. Oligodendrocyte fate after spinal cord injury. Neurotherapeutics 8(2), 262-273 (2011).

6 Cao Q, He Q, Wang Yet al. Transplantation of ciliary neurotrophic factor-expressing adult oligodendrocyte precursor cells promotes remyelination and functional recovery after spinal cord injury. J. Neurosci. 30(8), 29893001 (2010).

7 Nistor GI, Totoiu MO, Haque N, Carpenter MK, Keirstead HS. Human embryonic stem cells differentiate into oligodendrocytes in high purity and myelinate after spinal cord transplantation. Glia 49(3), 385-396 (2005).

- Original paper describing AST-OPC1 differentiation protocol.

8 Alsanie WF, Niclis JC, Petratos S. Human embryonic stem cell-derived oligodendrocytes: protocols and perspectives. Stem Cells Dev. 22(18), 2459-2476 (2013).

-• Review comparing AST-OPC1 differentiation protocol to alternative oligodendrocyte progenitor cell differentiation methods.

9 Hu BY, Du ZW, Zhang SC. Differentiation of human oligodendrocytes from pluripotent stem cells. Nat. Protoc. 4(11), 1614-1622 (2009).

10 Keirstead HS, Nistor G, Bernal Get al. Human embryonic stem cell-derived oligodendrocyte progenitor cell transplants remyelinate and restore locomotion after spinal cord injury. J. Neurosci. 25(19), 4694-4705 (2005).

- First study demostrating efficacy of AST-OPC1 in a rat model of thoracic spinal cord injury.

11 Zhang YW, Denham J, Thies RS. Oligodendrocyte progenitor cells derived from human embryonic stem cells express neurotrophic factors. Stem Cells Dev. 15(6), 943-952 (2006).

12 Karimi-Abdolrezaee S, Eftekharpour E, Wang J, Morshead CM, Fehlings MG. Delayed transplantation of adult neural precursor cells promotes remyelination and functional neurological recovery after spinal cord injury. J. Neurosci. 26(13), 3377-3389 (2006).

13 Goldman S. Stem and progenitor cell-based therapy of the human central nervous system. Nat. Biotechnol. 23(7), 862-871 (2005).

14 Nakamura M, Okano H, Toyama Y, Dai HN, Finn TP, Bregman BS. Transplantation of embryonic spinal cord-derived neurospheres support growth of supraspinal projections and functional recovery after spinal cord injury in the neonatal rat. J. Neurosci. Res 81(4), 457-468 (2005).

- Additional in vitro profiling of AST-OPC1, including secretion profile.

$15 \mathrm{Xu}$ C, Inokuma MS, Denham Jet al. Feeder-free growth of undifferentiated human embryonic stem cells. Nat. Biotechnol. 19(10), 971-974 (2001).

16 Li Y, Powell S, Brunette E, Lebkowski J, Mandalam R. Expansion of human embryonic stem cells in defined serumfree medium devoid of animal-derived products. Biotechnol. Bioeng. 91(6), 688-698 (2005).

17 Hatch MN, Nistor G, Keirstead HS. Derivation of highpurity oligodendroglial progenitors. Methods Mol. Biol. 549, 59-75 (2009).

18 Hulsebosch CE, Xu GY, Perez-Polo JR, Westlund KN, Taylor CP, Mcadoo DJ. Rodent model of chronic central pain after spinal cord contusion injury and effects of gabapentin. J. Neurotrauma 17(12), 1205-1217 (2000).

19 Wright MC, Mi R, Connor Eet al. Novel roles for osteopontin and clusterin in peripheral motor and sensory axon regeneration. J. Neurosci. 34(5), 1689-1700 (2014).

20 Klimaschewski L, Obermuller N, Witzgall R. Regulation of clusterin expression following spinal cord injury. Cell Tissue Res. 306(2), 209-216 (2001).

21 Tang SK, Knobloch RA, Maucksch C, Connor B. Redirection of doublecortin-positive cell migration by over-expression of the chemokines MCP-1, MIP-1alpha and Gro-alpha in the adult rat brain. Neuroscience 260, 240-248 (2014).

22 Gordon RJ, Mehrabi NF, Maucksch C, Connor B. Chemokines influence the migration and fate of neural precursor cells from the young adult and middle-aged rat subventricular zone. Exp. Neurol. 233(1), 587-594 (2012).

23 Zhang H, Chang M, Hansen CN, Basso DM, NobleHaeusslein LJ. Role of matrix metalloproteinases and therapeutic benefits of their inhibition in spinal cord injury. Neurotherapeutics 8(2), 206-220 (2011).

24 Wang R, Hong J, Lu Met al. Apoe mimetic ameliorates motor deficit and tissue damage in rat spinal cord injury. J. Neurosci. Res 92(7), 884-892 (2014).

25 Nothias JM, Mitsui T, Shumsky JS, Fischer I, Antonacci MD, Murray M. Combined effects of neurotrophin secreting transplants, exercise, and serotonergic drug challenge improve function in spinal rats. Neurorehabil. Neural Repair 19(4), 296-312 (2005).

26 Schnell L, Hunanyan AS, Bowers WJet al. Combined delivery of Nogo-A antibody, neurotrophin- 3 and the NMDA-NR2D subunit establishes a functional 'detour' in the hemisected spinal cord. Eur. J. Neurosci. 34(8), 1256-1267 (2011).

27 Mitsui T, Shumsky JS, Lepore AC, Murray M, Fischer I. Transplantation of neuronal and glial restricted precursors into contused spinal cord improves bladder and motor functions, decreases thermal hypersensitivity, and modifies intraspinal circuitry. J. Neurosci. 25(42), 9624-9636 (2005) 
28 Faulkner J, Keirstead HS. Human embryonic stem cellderived oligodendrocyte progenitors for the treatment of spinal cord injury. Transpl. Immunol. 15(2), 131-142 (2005).

29 Davies SJ, Shih CH, Noble M, Mayer-Proschel M, Davies JE, Proschel C. Transplantation of specific human astrocytes promotes functional recovery after spinal cord injury. PLoS ONE 6(3), e17328 (2011).

30 Vadivelu S, Stewart TJ, Qu Yet al. $\mathrm{Ng} 2+$ progenitors derived from embryonic stem cells penetrate glial scar and promote axonal outgrowth into white matter after spinal cord injury. Stem Cells Transl. Med. 4(4), 401-411 (2015).

31 Lu P, Woodruff G, Wang Yet al. Long-distance axonal growth from human induced pluripotent stem cells after spinal cord injury. Neuron 83(4), 789-796 (2014).

32 Noble M, Davies JE, Mayer-Proschel M, Proschel C, Davies SJ. Precursor cell biology and the development of astrocyte transplantation therapies: lessons from spinal cord injury. Neurotherapeutics 8(4), 677-693 (2011).

- Review of neural progenitor based therapies for spinal cord injury.

33 Douvaras P, Wang J, Zimmer Met al. Efficient generation of myelinating oligodendrocytes from primary progressive multiple sclerosis patients by induced pluripotent stem cells. Stem Cell Rep. 3(2), 250-259 (2014).

- Alternative oligodendrocyte diffentiation protocol using small molecules and extended differentiation time to produce mature oligodendrocytes.

34 Hu Z, Li T, Zhang X, Chen Y. Hepatocyte growth factor enhances the generation of high-purity oligodendrocytes from human embryonic stem cells. Differentiation 78(2-3), 177-184 (2009).

35 Sharp J, Frame J, Siegenthaler M, Nistor G, Keirstead HS. Human embryonic stem cell-derived oligodendrocyte progenitor cell transplants improve recovery after cervical spinal cord injury. Stem Cells 28(1), 152-163 (2010).

36 Ma Z, Cao Q, Zhang Let al. Oligodendrocyte precursor cells differentially expressing nogo-a but not mag are more permissive to neurite outgrowth than mature oligodendrocytes. Exp. Neurol. 217(1), 184-196 (2009).

37 Behrmann DL, Bresnahan JC, Beattie MS, Shah BR. Spinal cord injury produced by consistent mechanical displacement of the cord in rats: behavioral and histologic analysis. J. Neurotrauma 9(3), 197-217 (1992).
38 Metz GA, Curt A, Van De Meent H, Klusman I, Schwab ME, Dietz V. Validation of the weight-drop contusion model in rats: a comparative study of human spinal cord injury. J. Neurotrauma 17(1), 1-17 (2000).

39 Scheff SW, Rabchevsky AG, Fugaccia I, Main JA, Lumpp JE Jr. Experimental modeling of spinal cord injury: characterization of a force-defined injury device. J. Neurotrauma 20(2), 179-193 (2003).

40 Amariglio N, Hirshberg A, Scheithauer BWet al. Donor-derived brain tumor following neural stem cell transplantation in an ataxia telangiectasia patient. PLoS Med. 6(2), e1000029 (2009).

41 Cyranoski D. Strange lesions after stem-cell therapy. Nature 465(7301), 997 (2010).

42 Lee AS, Tang C, Cao Fet al. Effects of cell number on teratoma formation by human embryonic stem cells. Cell Cycle 8(16), 2608-2612 (2009).

43 Gropp M, Shilo V, Vainer Get al. Standardization of the teratoma assay for analysis of pluripotency of human es cells and biosafety of their differentiated progeny. PLoS ONE 7(9), e45532 (2012).

44 Werbowetski-Ogilvie TE, Bosse M, Stewart Met al. Characterization of human embryonic stem cells with features of neoplastic progression. Nat. Biotechnol. 27(1), 91-97 (2009).

45 Doi D, Morizane A, Kikuchi Tet al. Prolonged maturation culture favors a reduction in the tumorigenicity and the dopaminergic function of human ESC-derived neural cells in a primate model of Parkinson's disease. Stem Cells 30(5), 935-945 (2012).

46 Kriks S, Shim JW, Piao Jet al. Dopamine neurons derived from human es cells efficiently engraft in animal models of parkinson's disease. Nature 480(7378), 547-551 (2011).

47 Roy NS, Cleren C, Singh SK, Yang L, Beal MF, Goldman SA. Functional engraftment of human es cell-derived dopaminergic neurons enriched by coculture with telomerase-immortalized midbrain astrocytes. Nat. Med. 12(11), 1259-1268 (2006).

48 Breitbach M, Bostani T, Roell Wet al. Potential risks of bone marrow cell transplantation into infarcted hearts. Blood 110(4), 1362-1369 (2007). 